\title{
ARTICLE \\ Kinetics and mechanisms of mitotic inheritance of DNA methylation and their roles in aging-associated methylome deterioration
}

\author{
Xuan Ming $\mathbb{i D}^{1,2,3}$, Zhuqiang Zhang ${ }^{2}$, Zhuoning Zou ${ }^{4}$, Cong $\mathrm{Lv}^{5}$, Qiang Dong ${ }^{2}$, Qixiang $\mathrm{He}^{4}$, Yangyang $\mathrm{Yi}^{2,4}$, Yingfeng $\mathrm{Li}^{2}$, \\ Hailin Wang ${ }^{5}$ and Bing Zhu ${ }^{2,4}$
}

\begin{abstract}
Mitotic inheritance of the DNA methylome is a challenging task for the maintenance of cell identity. Whether DNA methylation pattern in different genomic contexts can all be faithfully maintained is an open question. A replication-coupled DNA methylation maintenance model was proposed decades ago, but some observations suggest that a replication-uncoupled maintenance mechanism exists. However, the capacity and the underlying molecular events of replication-uncoupled maintenance are unclear. By measuring maintenance kinetics at the single-molecule level and assessing mutant cells with perturbation of various mechanisms, we found that the kinetics of replication-coupled maintenance are governed by the UHRF1-Ligase 1 and PCNADNMT1 interactions, whereas nucleosome occupancy and the interaction between UHRF1 and methylated H3K9 specifically regulate replication-uncoupled maintenance. Surprisingly, replication-uncoupled maintenance is sufficiently robust to largely restore the methylome when replication-coupled maintenance is severely impaired. However, solo-WCGW sites and other CpG sites displaying aging- and cancer-associated hypomethylation exhibit low maintenance efficiency, suggesting that although quite robust, mitotic inheritance of methylation is imperfect and that this imperfection may contribute to selective hypomethylation during aging and tumorigenesis.
\end{abstract}

Cell Research (2020) 30:980-996; https://doi.org/10.1038/s41422-020-0359-9

\section{INTRODUCTION}

DNA methylation is an epigenetic modification involved in longterm gene silencing, which is essential for cell type-specific gene expression pattern regulation, $\mathrm{X}$ chromosome inactivation and repetitive element repression. ${ }^{1,2}$ In each round of mitotic cell division, DNA replication leaves the pre-existing methylated CpGs in a hemimethylated state with the newly synthesized strand unmethylated. Ensuring the restoration of the DNA methylome before cells enter the next $S$ phase is critical for cell identity maintenance. ${ }^{3}$ However, the kinetics, underlying mechanisms and fidelity of the post-replication DNA methylation maintenance process are not well studied.

The kinetics of DNA methylation maintenance has been debated for decades. Some studies suggested that methylation maintenance was achieved within minutes of DNA replication, ${ }^{4-7}$ but some other studies observed several hours of delay after replication..$^{8-11}$ However, the methylation status of the parent and the daughter strands have never been measured at the single-molecule level, which is required for the precise calculation of the maintenance ratio and the evaluation of methylation maintenance kinetics.

Importantly, DNA methylation maintenance in vivo occurs in a very complex environment, and many chromatin events, such as nucleosome occupancy and histone modifications, likely participate in the regulation of maintenance kinetics. However, it remains unclear whether DNA methylation maintenance kinetics differs in different genomic regions and sequence contexts.

DNMT1 is the main enzyme responsible for methylation maintenance, ${ }^{12}$ and it prefers hemimethylated substrate in vitro. ${ }^{13-18} \mathrm{We}$ recently reported the de novo methylation activity of DNMT1 in oocytes without DNA replication, ${ }^{19}$ but whether DNMT1 can perform de novo methylation during replication-coupled methylation maintenance is currently unknown.

Most studies regarding mechanisms regulating DNA methylation maintenance were based on biochemical and genetic experiments, which provided plenty of knowledge about DNA methylation maintenance. ${ }^{20}$ However, genetic data obtained by measuring DNA methylation level in mutant cells often do not reconcile with biochemical results, because the steady state level of DNA methylation only provides the end-point information, but not the kinetics, in which these biochemical mechanisms may play a role. PCNA was reported to bind and recruit DNMT1 to replication forks, ${ }^{21}$ but later studies showed that this interaction was not essential for maintaining the DNA methylation level. ${ }^{22,23}$ UHRF1 connects DNA methylation maintenance with nucleosome

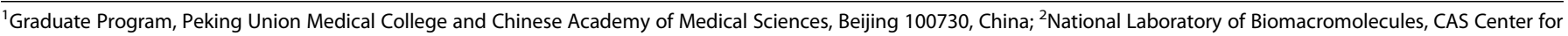

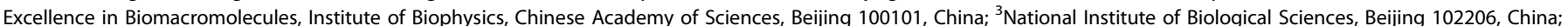

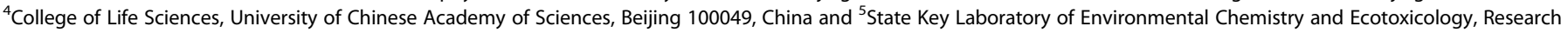
Center for Eco-Environmental Sciences, Chinese Academy of Sciences, Beijing 100085, China

Correspondence: Bing Zhu (zhubing@ibp.ac.cn)

These authors contributed equally: Xuan Ming, Zhuqiang Zhang

Received: 2 February 2020 Accepted: 27 May 2020

Published online: 24 June 2020 
modifications. ${ }^{24}$ The UHRF1 TTD domain preferentially binds $\mathrm{H} 3 \mathrm{~K} 9 \mathrm{me} 2 / 3,{ }^{25,26}$ but mutations disrupting this interaction only cause a minor reduction in the DNA methylation level. ${ }^{27}$ Thus, the exact functional contributions of these biochemical mechanisms need to be determined, and a full model elucidating the regulators governing replication-coupled maintenance and the potentially existing replication-uncoupled maintenance awaits to be revealed.

On the other hand, although DNA methylation is believed to be the most faithful epigenetic mark inherited during mitotic division, global hypomethylation in certain selective genomic contexts is observed in aging and tumorigenesis. ${ }^{28-33}$ In fact, recent studies indicate that DNA methylation level is a promising biomarker for age prediction. ${ }^{34-36}$ However, the underlying mechanism of methylation loss in aging and tumorigenesis is unclear. ${ }^{37-39}$

To address the aforementioned questions, we developed Hammer-seq (Hairpin-assisted mapping of methylation of replicated DNA), which can examine the dynamic DNA methylation maintenance process at the single-molecule level with high spatiotemporal resolution. First, we observed that methylation maintenance can be divided into two phases, a rapid replicationcoupled phase within minutes of fork passage and a gradual replication-uncoupled phase afterwards. By perturbing various mechanisms, we found that the UHRF1-Ligase1 and PCNADNMT1 interactions contribute to replication-coupled rapid maintenance, whereas $\mathrm{H} 3 \mathrm{~K} 9 \mathrm{me} 2 / 3$ and nucleosome occupancy specifically regulate replication-uncoupled maintenance. We also observed a surprisingly strong backup effect of replicationuncoupled maintenance that can largely rescue the failure of replication-coupled maintenance. Overall, mitotic inheritance of DNA methylation involves a robust replication-coupled maintenance system and a highly potent replication-uncoupled maintenance backup system. Intriguingly, methylation maintenance remains to be imperfect. CpGs in certain sequence contexts, such as solo-WCGW CpGs (CpG sites immediately flanked by A/T bases and with no neighboring $\mathrm{CpG}$ sites within $70 \mathrm{bp}){ }^{40}$ displayed the slowest maintenance kinetics. Importantly, these sequence contexts match those reported to exhibit selective methylation loss during aging and tumorigenesis. Thus, the inevitable failure of methylation maintenance in these sequence contexts is an important contributor to methylome deterioration during aging and tumorigenesis.

\section{RESULTS}

Hammer-seq delineates the methylation maintenance kinetics of replicated DNA

Simultaneous detection of the methylation status of newly replicated parent and daughter DNA strands at the singlemolecule level is essential for the precise measurement of the maintenance kinetics and for ruling out any potential averaging effect of bulk measurements. Therefore, we developed a new method, termed Hammer-seq, which combines EdU labeling, biotin conjugation, immunopurification and hairpin bisulfite sequencing technologies (Fig. 1a). ${ }^{41,42}$

Replication forks fire at different time within $\mathrm{S}$ phase, which is a challenge for accurately measuring maintenance kinetics. To avoid mixing kinetics information from regions replicated at different time, DNA replicated within the time window of $4 \mathrm{~min}$ were labeled with EdU, and these replicated DNA were specifically purified for measuring their methylation maintenance kinetics (Fig. 1a).

We were able to pulse label HeLa cells with EdU for durations as short as 4 min to capture newly synthesized DNA (Supplementary information, Fig. S1a), and paired-end hairpin bisulfite sequencing allowed us to simultaneously measure the methylation status of parent and daughter strands at the single-molecule level with single-base resolution (Supplementary information, Fig. S1b).
Hammer-seq displayed strong enrichment of newly replicated regions (Supplementary information, Fig. S1c) and successfully captured maintenance intermediates (Supplementary information, Fig. S1b). We chased for various time points up to $24 \mathrm{~h}$ and performed Hammer-seq to measure maintenance kinetics during and after $\mathrm{S}$ phase (Fig. 1a and Supplementary information, Fig. S2a)

For each paired sequencing read, we monitored the methylation status of CpGs in each strand individually. Reads containing no methylated $\mathrm{CpG}$ sites in either strand were excluded from analysis. Reads displaying equal methylation levels in the two strands were considered to have completed maintenance. For reads displaying unequal methylation levels in the two strands, we arbitrarily assigned the strand with the higher methylation level as the parent strand and the strand with the lower methylation level as the daughter strand. This arbitrary assignment likely underestimated the maintenance efficiency at later time points but remained highly rational at the global scale because the assigned daughter strands displayed methylation levels similar to those of the EdU-labeled daughter strands measured in experiments without hairpin sequencing (Supplementary information, Fig. S1d).

We defined the number of methylated CpGs in the parent strand as "total mCpGs" and the number of CpGs methylated in both strands as "maintained mCpGs" and then calculated the maintenance ratio of regions of interest using the following equation: maintenance ratio $(\%)=$ maintained $\mathrm{mCpGs} /$ total $\mathrm{mCpGs} \times 100 \%$.

The maintenance ratios determined in two independent biological replicates were highly reproducible, demonstrating the robustness of Hammer-seq (Supplementary information, Fig. S3 and Table S1).

Maintenance methylation was indeed highly efficient, because even without chasing, the maintenance ratio was greater than $50 \%$ (Fig. 1b), indicating that more than half of the methylated CpGs were successfully maintained within 4 min of fork passage. However, although more than $80 \%$ of the methylated CpGs were fully maintained within $30 \mathrm{~min}$, it took approximately $10 \mathrm{~h}$ to finish the maintenance process at the remaining sites (Fig. 1b). Our results in HeLa cells supported the previous observation made in human ES cells regarding a broad delay in maintenance, ${ }^{11}$ but contradicted the observation in the other recent study in human ES cells, which reported no apparent difference between parent and daughter strand methylation 20 min after fork passage. ${ }^{7}$ Thus, although methylation can be robustly maintained within minutes of fork passage (we termed as replication-coupled maintenance phase), methylation maintenance continued across the rest of cell cycle (we termed as replication-uncoupled maintenance phase) and it also contributes to ensure efficient inheritance of the DNA methylome.

Differential maintenance kinetics in distinct genomic contexts After obtaining the global maintenance kinetics, we asked whether distinct genomic regions share similar maintenance kinetics. Therefore, we determined the maintenance kinetics at various genomic features, including various repetitive sequences, CpG islands, promoters, enhancers, etc. Interestingly, among different repeats, low-complexity repeats displayed the fastest maintenance kinetics, whereas LINEs and LTRs displayed the slowest maintenance kinetics (Fig. 1c). In addition, CpG islands, promoters and exons displayed faster maintenance kinetics than introns, intergenic regions and enhancers (Fig. 1d). CpG islands are known as hypomethylated regions in many cell types, but certain cancer cells, including HeLa cells, exhibit substantially elevated DNA methylation levels at CpG islands, although their bulk methylation levels are reduced (Supplementary information, Fig. S1e). ${ }^{39,43}$

We asked which molecular signature could potentially be responsible for the above observation. DNMT1 is a processing enzyme. ${ }^{44-46}$ Therefore, its catalysis might be regulated by the 


\section{a}

Cell cycle synchronization

(thymidine-aphidicolin block

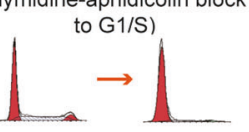

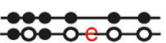

$\because-1$ Biotin conjugation

$\stackrel{\text { (Click-it reaction) }}{\longrightarrow}$

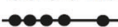

:\%-

$\because: 0 \div-$

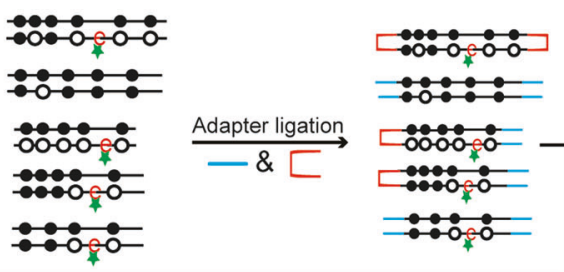

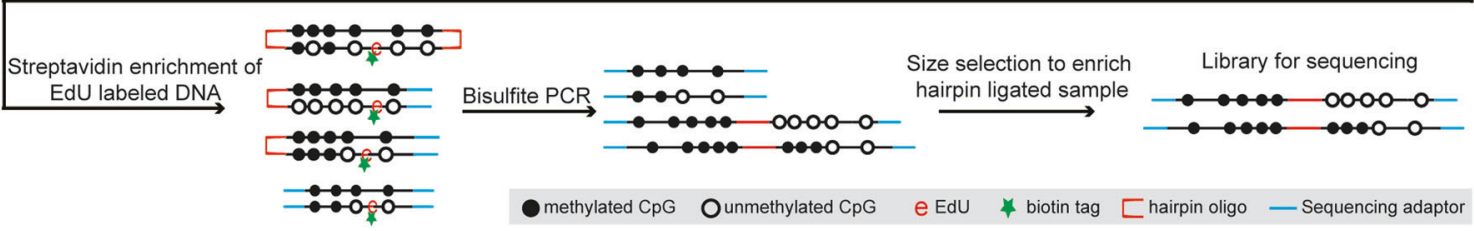

b

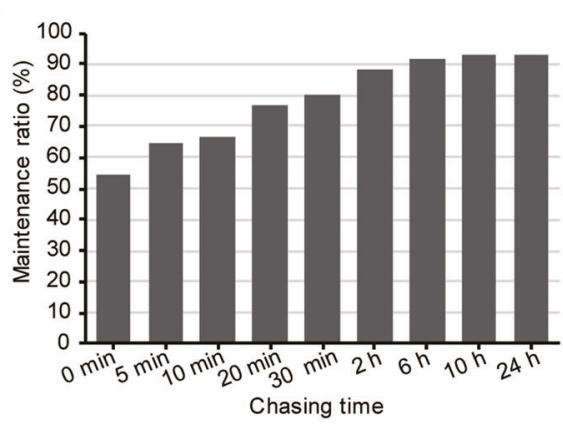

e

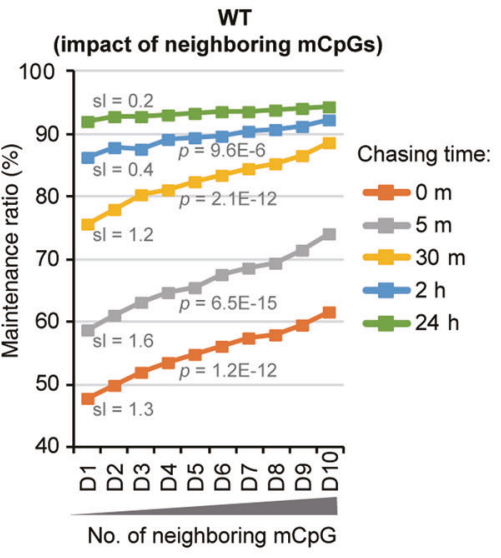

C

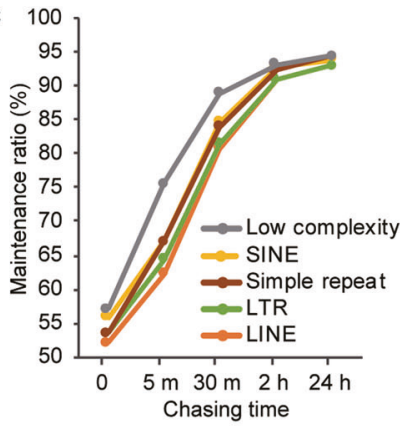

d

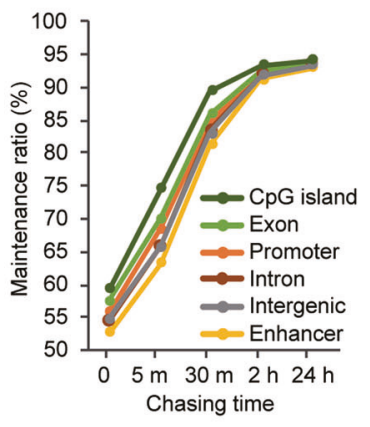

f

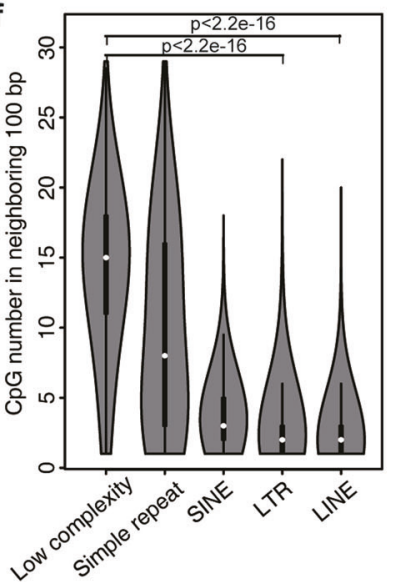

g

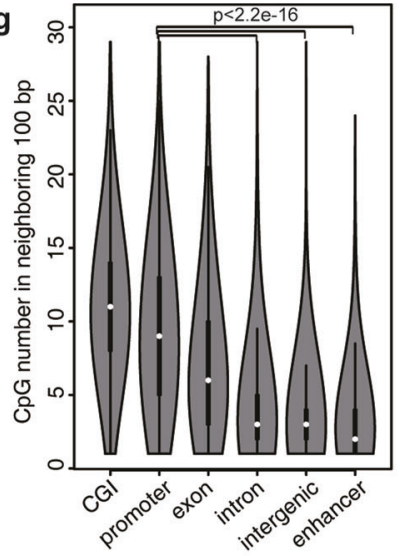

h

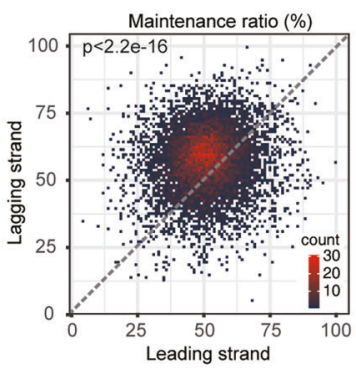

i

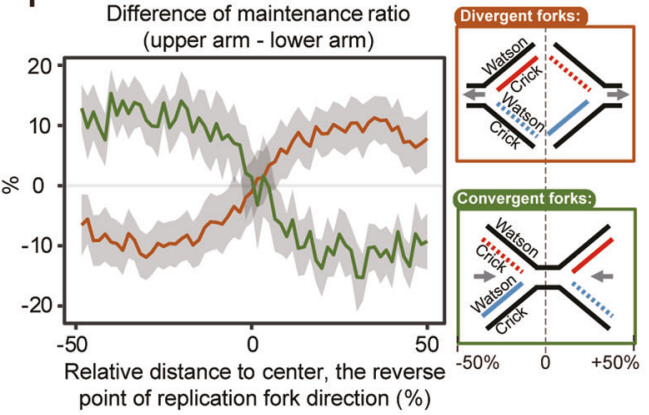

j

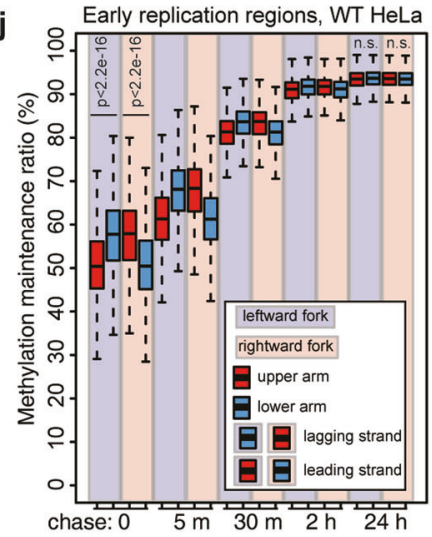

number of nearby methylated CpGs. Indeed, regions with lower CpG methylation levels or CpG densities displayed slower maintenance rates (Supplementary information, Fig. S1f), and the number of neighboring methylated $\mathrm{CpGs}$ exhibited a markedly positive effect on maintenance kinetics (Fig. 1e).
Consistently, among repeats, LINEs and LTRs had the lowest CpG density, whereas low-complexity repeats had the highest CpG density (Fig. 1f); in addition, CpG islands and promoters had a higher $\mathrm{CpG}$ density than introns, intergenic regions and enhancers (Fig. 1g). 
Fig. 1 Hammer-seq delineates the kinetics of methylation maintenance. a Flowchart of Hammer-seq. b Global kinetics of methylation maintenance in early S domains in wild-type (WT) HeLa cells. Global maintenance ratios are shown as the maintained mCpGs/total mCpGs for each chasing time point. c Kinetics of DNA methylation maintenance for five classes of repeat elements in WT HeLa cells. The maintenance ratio was calculated based on individual CpG sites located in each class of repeats, and the average values of all CpG sites are shown for all time points. d Kinetics of DNA methylation maintenance in different genomic regions in WT HeLa cells. e Maintenance kinetics across CpG groups with different numbers of neighboring methylated CpGs (mCpGs) in WT cells. All covered CpG sites were divided into 10 ranges according to deciles of the number of $\mathrm{mCpGs}$ within $100 \mathrm{bp}$ (centered on CpG sites), and the average maintenance ratio of each group was plotted. The $\mathrm{mCpG}$ numbers are the sum of the methylation levels of every CpG in the range. "sl" indicates the slope of the linear regression of the curve, and a slope value of 0.1 means $1 \%$ changes across all 10 intervals. Datasets from replicates are pooled. $P$ values are from comparison of slope for chase $24 \mathrm{~h}$ to the other four time points. $\mathrm{f}$ Violin plots showing the $\mathrm{CpG}$ number in the neighboring $100 \mathrm{bp}$ for $\mathrm{CpG}$ sites located in five classes of repeat elements. g Violin plots showing the CpG number in the neighboring $100 \mathrm{bp}$ for CpG sites located in different genomic regions. $\mathbf{h}$ Scatter plot showing the maintenance ratio of the leading strand and lagging strand at the 0 min chase time point. Domains with strong replication fork directions were selected, and reads located in the upper or lower arm were used to determine the maintenance ratio for the leading or lagging strand, respectively. Each dot indicates a part of the replication domain with a strong replication direction bias (overlap with high RFD score regions; RFD, replication fork direction; see the Materials and methods section). i Differences in the maintenance ratio between the upper arm and lower arm of the replication fork around genomic regions where replication forks converge (green) or diverge (bronze). Convergent forks are regions where the RFD scores transition from positive (rightward) to negative (leftward), while divergent forks are regions with the opposite transition. Reads located in the upper arm or lower arm were used to calculate the maintenance ratio of each arm in 50 equal bins, and the difference between matched bins is plotted. Datasets of the 0 min chase time point in WT cells are used. In the legend, the colored solid lines indicate the leading strand, while the colored dashed lines indicate the lagging strand. The gray arrows indicate replication fork directions. Shade indicates the standard error of the mean. $\mathbf{j}$ Maintenance kinetics of the leading strand and lagging strand. For leftward domains and rightward domains, the maintenance ratios were determined by reads located in the upper and lower arms, respectively. The leading and lagging strands are discriminated as shown in Fig. $1 \mathrm{i}$.

Considering the above findings, we concluded that maintenance kinetics differ in different genomic contexts and that the neighboring methylated $\mathrm{CpG}$ density is a very important regulator of maintenance kinetics.

Faster maintenance kinetics in the lagging strand

Recent studies have reported that parental histones and new histones are differentially distributed to the leading strand and lagging strand. ${ }^{47,48}$ This difference prompted us to ask whether DNA methylation maintenance kinetics are also different between the leading and lagging strands. Although mammalian cells display heterogeneity in the usage of replication origins, certain replication origins are preferentially used in the majority of cells, and replication fork directionality (RFD) has been previously defined by OK-seq in HeLa cells. ${ }^{49}$ Thus, we allocated Hammer-seq reads to the leading and lagging strands (see the Materials and methods section for details) and calculated the maintenance ratios of the leading and lagging strands individually. Obviously, at the 0 min chase time point, DNA methylation maintenance occurred faster in lagging strands than in leading strands (Fig. 1h, i). The difference in the maintenance ratio between the leading and lagging strands gradually decreased and could no longer be discriminated at the $24 \mathrm{~h}$ chase time point (Fig. 1j).

The DNMT1-PCNA and UHRF1-Ligase1 interactions contribute to rapid methylation maintenance in the replication-coupled phase The basic machinery for maintenance includes the hemimethylated CpG binding activity of the UHRF1 SRA domain, ${ }^{50,51}$ the histone $\mathrm{H} 3$ tail binding activity of the UHRF1 PHD domain, ${ }^{52-55}$ and the $\mathrm{H} 3$ ubiquitination activity of the UHRF1 RING domain, ${ }^{56}$ which stimulates the catalytic activity of DNMT1. ${ }^{57,58}$ Impairing these components reduces bulk DNA methylation, ${ }^{20}$ indicating that these mechanisms not only affect maintenance kinetics but also affect the endpoint. However, disruption of the DNMT1-PCNA or UHRF1-H3K9me2/3 interaction does not significantly affect bulk methylation, ${ }^{22,23,27}$ while the mechanism is not well understood. Because Hammer-seq allows us to measure the maintenance kinetics in a single cell cycle, we decided to combine various mutant cell lines to investigate the precise roles of distinct mechanisms potentially involved in regulating DNA methylation.

DNMT1 interacts with PCNA and mutating histidine 170 of DNMT1 to valine disrupts the interaction between DNMT1 and PCNA. ${ }^{21}$ To elucidate the functional significance of this interaction interface, we generated a DNMT1-H170V homozygous ( 3 alleles in HeLa cells) knock-in mutant cell line (Supplementary information, Fig. S2b and Table S2) and confirmed its largely normal bulk methylation level (Fig. 2a and Supplementary information, Fig. S2c). We then measured its maintenance kinetics (Fig. 2b). Interestingly, at the $0 \mathrm{~min}$ chase time point, this mutant displayed a significantly reduced maintenance ratio (reduced from $54 \%$ for the WT to $42 \%$ ), but the defect was quickly compensated after fork passage (Fig. 2b). These results revealed a role for the DNMT1PCNA interaction in regulating replication-coupled maintenance and explained why its impairment had little effect on the bulk methylation level, because replication-uncoupled maintenance was capable of backing up a defective replication-coupled maintenance system.

The tudor domain of UHRF1 specifically recognizes methylated H3K9. ${ }^{25,26}$ Given that the H3K9-specific dimethylases G9a/GLP associate with the replication fork, ${ }^{59}$ the interaction between UHRF1 and H3K9 methylation was proposed to regulate replication-coupled maintenance. ${ }^{60}$ To test this idea, we generated a homozygous ( 3 alleles in HeLa cells) knock-in cell line with mutation of the residues of the UHRF1 tudor domain that interact with H3K9 methylation (UHRF1 Y188V/Y191V, termed UHRF1-TTD) (Supplementary information, Fig. S4a). ${ }^{26,27}$ This mutant displayed substantially delayed maintenance in replication-coupled and replication-uncoupled phases (Fig. 2c). Interestingly, although the UHRF1-TTD mutant exhibited much more delayed kinetics than the DNMT1-H170V mutant, methylation maintenance was largely completed in $24 \mathrm{~h}$, ensuring nearly complete recovery of global methylation in one cell cycle (Fig. 2c), which explained why impairing the UHRF1 tudor domain only caused a minor reduction in global methylation (Fig. 2a and Supplementary information, Fig. S2c). ${ }^{27}$ This finding again demonstrated that replicationuncoupled maintenance was capable of backing up a defective replication-coupled maintenance system.

Given the role of the UHRF1 tudor domain in recognizing H3K9 methylation, we asked whether H3K9 methylation regulates maintenance. We categorized all CpG sites into 10 divisions based on the level of $\mathrm{H} 3 \mathrm{~K} 9 \mathrm{me} 2$ or $\mathrm{H} 3 \mathrm{~K} 9 \mathrm{me} 3$ within the neighboring $1 \mathrm{~kb}$ region and analyzed the maintenance ratio of each division. Surprisingly, CpG sites within regions with higher H3K9 methylation levels did not display higher maintenance ratios at any of the time points (Fig. 3a, left panel and Supplementary information, Fig. S4b). These results implied that H3K9 methylation contributed little to maintenance, which did not seem to be consistent with 
a Early replicating regions, chase $24 \mathrm{hr}$, parent strand

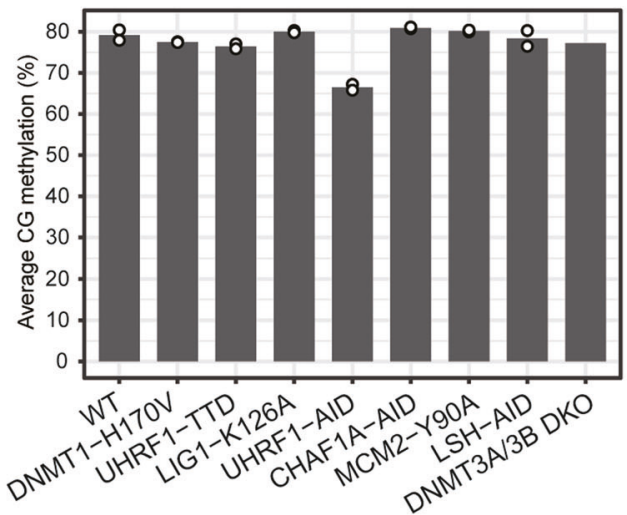

b

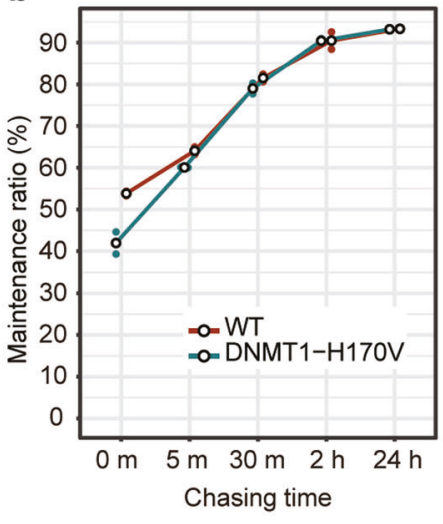

C

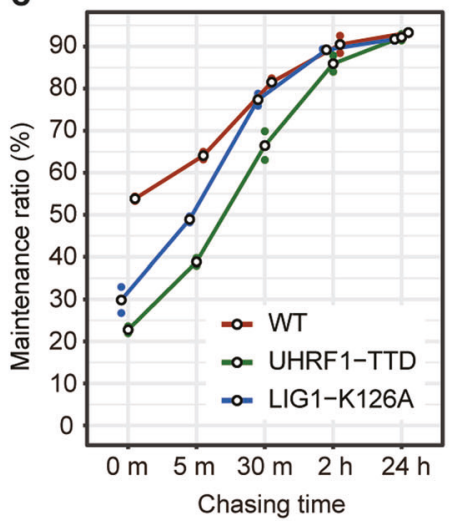

Fig. 2 The UHRF1-Ligase 1 and DNMT1-PCNA interactions contribute to rapid maintenance methylation in the DNA replication-coupled phase. a DNA methylation levels of parent strands in WT HeLa and various mutant cell lines. Circles show the values from all replicates and the columns indicate the average of replicates. b Comparison of maintenance kinetics between DNMT1-H170V mutant and WT cells. Colored dots show the global maintenance ratios of all replicates and the circles indicate the average of replicates. c Comparison of maintenance kinetics among UHRF1-TTD, LIG1-K126A and WT cells. Symbols are used similarly to those in $\mathbf{b}$.

the apparent importance of the UHRF1 tudor domain (Fig. 2c) and was puzzling and needed clarification.

Interestingly, the UHRF1 tudor domain also associates with DNA ligase 1 (LIG1), which is responsible for sealing Okazaki fragments, in a LIG1 K126 methylation-dependent manner because residues surrounding LIG1 K126 are highly similar to residues surrounding H3K9; and because LIG1 K126 is methylated by G9a/GLP and recognized by the UHRF1 tudor domain via the same binding pocket that recognizes methylated $\mathrm{H} 3 \mathrm{~K} 9$, and disrupting UHRF1/ LIG1 interaction can reduce UHRF1 recruitment to replication fork. ${ }^{61}$ Therefore, we reasoned that the defective kinetics of UHRF1-TTD mutant cells were due to the dissociation of UHRF1 from methylated LIG1 K126 rather than methylated H3K9. To evaluate this hypothesis, we generated a homozygous ( 3 alleles in HeLa cells) knock-in cell line containing the LIG1 K126A mutant (Fig. 2a; Supplementary information, Figs. S2c and S4c). Indeed, at the 0 min chase time point, LIG1-K126A cells also displayed obvious maintenance defect, similar to UHRF1-TTD cells (Fig. 2c), indicating that replication-coupled maintenance was strongly compromised by disruption of the UHRF1-LIG1 interaction. However, the maintenance kinetics of LIG1-K126A and UHRF1TTD cells at later time points were quite different, and LIG1-K126A cells displayed much faster replication-uncoupled maintenance rate (Fig. 2c). These results suggest that the UHRF1 tudor domain has dual roles in regulating both replication-coupled and replication-uncoupled maintenance and that its primary binding partner in the replication-coupled phase is LIG1, a component of the replication machinery. However, in the replication-uncoupled phase, the UHRF1 tudor domain likely regulates maintenance via its interaction with other partners, most likely methylated H3K9.

The above data collectively demonstrate that replicationcoupled maintenance is facilitated by the interactions between methylation maintenance machinery and replication machinery, such as DNMT1-PCNA and UHRF1-LIG1.

Interaction between UHRF1 and H3K9me2 compensates for the intrinsic disadvantage of methylation maintenance in these regions

To validate the hypothesis that the interaction between the UHRF1 tudor domain and methylated H3K9 regulates replicationuncoupled maintenance, we analyzed the maintenance ratio of UHRF1-TTD cells at various time points for all divisions with different $\mathrm{H} 3 \mathrm{~K} 9 \mathrm{me} 2$ or $\mathrm{H} 3 \mathrm{~K} 9 \mathrm{me} 3$ levels. Indeed, in UHRF1-TTD cells, the maintenance ratio was negatively correlated with the level of $\mathrm{H} 3 \mathrm{~K} 9 \mathrm{me} 2$ at the $5 \mathrm{~min}, 30 \mathrm{~min}$ and $2 \mathrm{~h}$ chase time points but not at the $0 \mathrm{~min}$ and $24 \mathrm{~h}$ chase time points, and the differences between UHRF1-TTD and WT cells were statistically significant (Fig. 3a). The effect of H3K9me3 appeared to be much more modest (Supplementary information, Fig. S4d). Consistent with these results, LIG1-K126A cells only showed much more modest differences across the H3K9 methylation divisions (Supplementary information, Fig. S4e).

These intriguing observations led to the following conclusions: 1) H3K9 methylation has little effect on replication-coupled maintenance, which further suggests that the major partner of the UHRF1 tudor domain in the replication-coupled phase is methylated LIG1 K126 but not methylated H3K9; 2) in wild-type cells, DNA methylation is maintained with similar kinetics in all groups divided according to the H3K9 methylation level; and 3) regions enriched for $\mathrm{H} 3 \mathrm{~K} 9$ methylation are intrinsically disfavored for maintenance, and the interaction between UHRF1 and H3K9 methylation helps to alleviate this disadvantage.

Regions enriched for H3K9 methylation are disfavored for maintenance and need the interaction with the UHRF1 tudor domain as a remedy. These observations prompted us to ask what kinds of intrinsic characteristics associated with these regions may negatively regulate maintenance. Given the strong positive effect of the neighboring $\mathrm{mCpG}$ density in regulating maintenance kinetics (Fig. 1e), we examined whether regions enriched for H3K9me2 naturally contained fewer CpG sites and $\mathrm{mCpG}$ sites and found that this was the case (Fig. 3b). Importantly, when we fixed the number of neighboring $\mathrm{mCpG}$ sites, the maintenance ratios in regions with different $\mathrm{H} 3 \mathrm{~K} 9 \mathrm{me} 2$ levels were largely equalized in UHRF1-TTD cells (Fig. 3c). Because the 30 min time point displayed the most significant changes in the impact of $\mathrm{H} 3 \mathrm{~K} 9 \mathrm{me} 2$ between UHRF1-TTD and WT cells, we used this time point to analyze the impact of $\mathrm{H} 3 \mathrm{~K} 9 \mathrm{me} 2$ with fixed range of neighboring $\mathrm{mCpGs}$. We found that equalizing neighboring $\mathrm{mCpGs}$ decreased, and even diminish the differential effect of H3K9me2 in UHRF1-TTD and WT cells, respectively (Fig. $3 \mathrm{~d}$ ), and the statistical significances of difference between UHRF1-TTD and WT are also reduced for several order of magnitudes (Fig. 3e). In conclusion, regions enriched for $\mathrm{H} 3 \mathrm{~K} 9 \mathrm{me} 2$ are poor in total $\mathrm{mCpG}$ sites, and this intrinsic disadvantage is compensated by the interaction between the UHRF1 tudor domain and H3K9me2.

In addition, UHRF1-TTD cells displayed minor but reproducibly observed bulk DNA methylation loss (Fig. 2a and Supplementary information, Fig. S2c), ${ }_{i}^{27}$ thus, we compared the DNA methylation levels of the parent strands in UHRF1-TTD and WT cells and found that regions with higher levels of $\mathrm{H} 3 \mathrm{~K} 9 \mathrm{me} 2$ clearly had a greater 


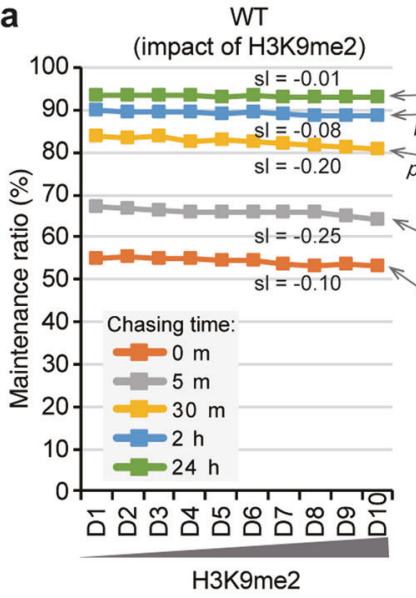

C neighboring $\mathrm{mCpG}$ No. between deciles of $40 \%$ and $50 \%$ )

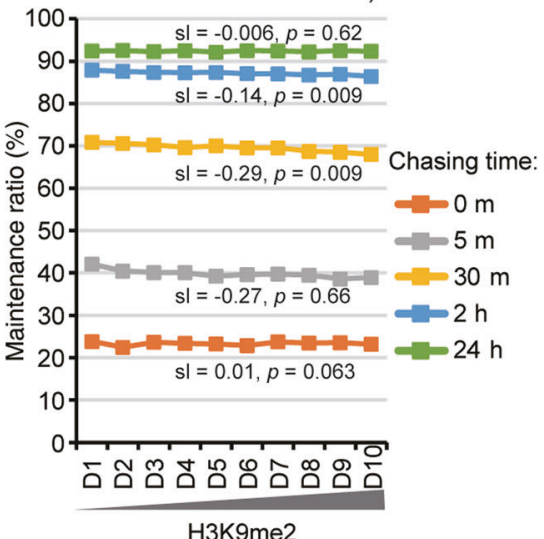

H3K9me2
UHRF1-TTD (impact of $\mathrm{H} 3 \mathrm{~K} 9 \mathrm{me} 2$ )
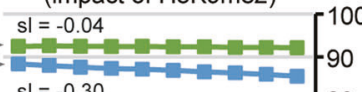

$\mathrm{sl}=-0.30 \quad-80$
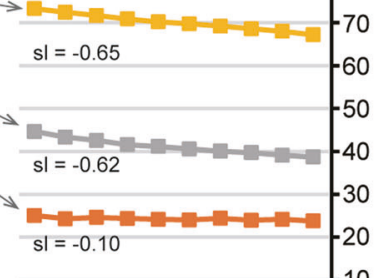

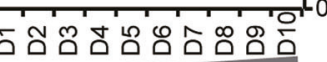
H3K9me2

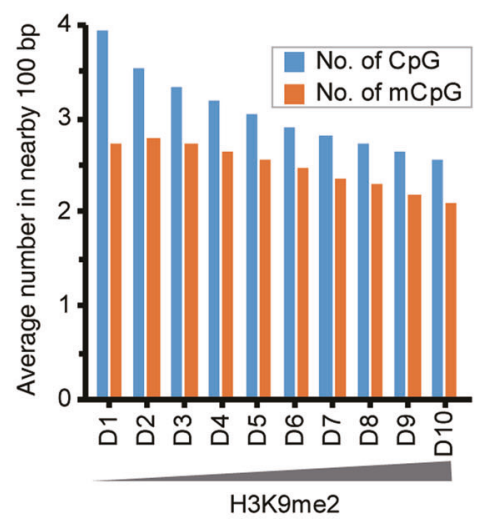

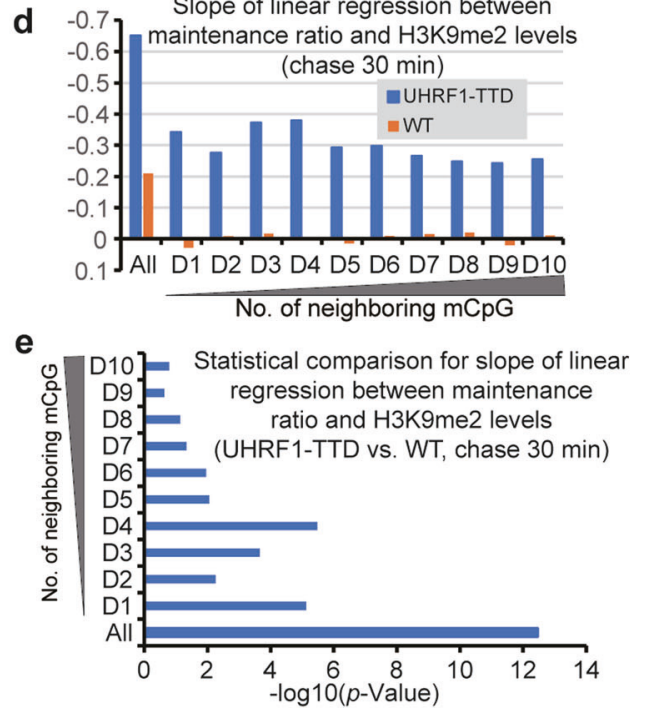

f

Methylation loss ( subtract UHRF1-TTD from WT)

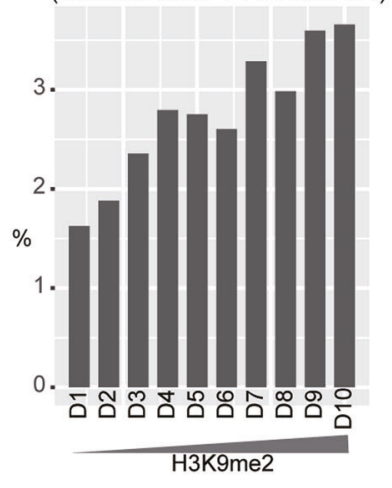

Fig. 3 Interaction between the UHRF1 tudor domain and H3K9me2 balances the intrinsic disadvantage of methylation maintenance in these regions. a Maintenance kinetics across $\mathrm{CpG}$ groups with different H3K9me2 levels (WT cells, left panel; UHRF1-TTD cells, right panel). All covered CpG sites were divided into 10 equal ranges according to deciles of their H3K9me2 level within $1 \mathrm{~kb}$ (centered on CpG sites), and the average maintenance ratio of each group was plotted in a similar way to Fig. 1e. $P$ values are calculated from ANOVA of slopes of corresponding time points. b Average number of neighboring CpGs and $\mathrm{mCpGs}$ within 100 bp across CpG groups with different H3K9me2 levels. c Similar plot to Fig. 3a, right panel, but with the number of neighboring $\mathrm{mCpGs}$ fixed to the level of the D5 group in Fig. 1 e. $P$ values are from comparison of slope of UHRF1-TTD to WT at each time point. $\mathbf{d}$ Linear repression slopes between maintenance ratio and ten ranges of H3K9me2 levels in WT and UHRF1-TTD cells (chase $30 \mathrm{~min}$ ). All CpGs and CpGs with fixed range of neighboring mCpGs are plotted for comparison. e Statistical comparison of slopes in $\mathbf{d}$ between UHRF1-TTD and WT for all CpGs and CpGs with fixed range of neighboring $\mathrm{mCpGs.} \mathbf{f}$ Differences in methylation levels between WT and UHRF1-TTD cells across CpG groups with different $\mathrm{H} 3 \mathrm{~K} 9 \mathrm{me} 2$ levels. Parent strands at the $24 \mathrm{~h}$ chase time point were used to determine the methylation level in UHRF1-TTD mutant and WT cells.

extent of DNA methylation loss (Fig. 3f). Therefore, although regions enriched for $\mathrm{H} 3 \mathrm{~K} 9 \mathrm{me} 2$ exhibited nearly complete recovery of methylation levels in one cell cycle (Fig. 3a, right panel), subtle losses inevitably occurred and accumulated after additional rounds of cell division.

Potent capacity of replication-uncoupled maintenance At the 0 min chase time, the maintenance ratio of DNMT1-H170V mutant cells was $42 \%$, compared to the $54 \%$ of the WT cells, but 30 min later, the maintenance ratio of DNMT1-H170V cells was at $80 \%$ maintenance ratio, similar to that of WT cells (Fig. 2b). LIG1K126A mutant cells displayed a more severe replication-coupled maintenance defect; at the 0 min chase time, only $29 \%$ of maintenance was accomplished; however, $2 \mathrm{~h}$ later, the maintenance ratio of LIG1-K126A cells was $89 \%$, similar to that of WT cells (Fig. 2c). These observations suggest that the replicationuncoupled maintenance system likely has a very strong capacity to address the potential failure of replication-coupled maintenance that is not in use in normal contexts.

To measure this capacity, we applied the auxin-based inducible and reversible protein degradation system ${ }^{62,63}$ and generated an UHRF1-AID cell line with knock-in of an auxin-inducible degron (AID) tag in all the endogenous UHRF1 alleles (3 alleles in HeLa cells) (Supplementary information, Fig. S5a). Although knock-in cells displayed moderately reduced UHRF1 expression (Supplementary information, Fig. S5a) and moderately reduced global DNA methylation (Fig. 2a), the DNA methylation level remained sufficiently high for analysis of methylation maintenance kinetics. We arrested UHRF1-AID cells at the G1/S phase boundary and induced UHRF1 degradation with auxin (IAA) prior to pulsing (Supplementary information, Fig. S5b). As expected, degradation of UHRF1 severely impaired both replication-coupled and replication-uncoupled maintenance (Fig. 4a) to a greater extent than all other mutants tested earlier. Notably, a limited level of 


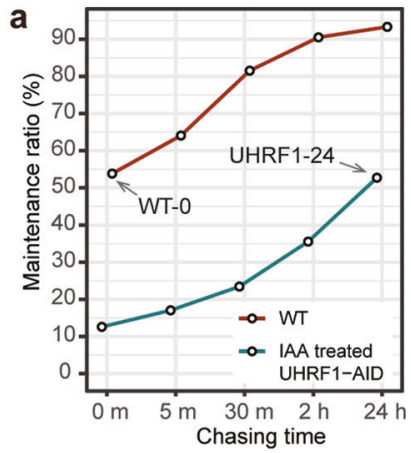

b

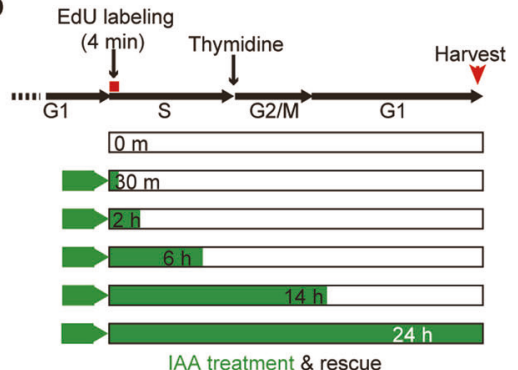

IAA treatment \& rescue
C

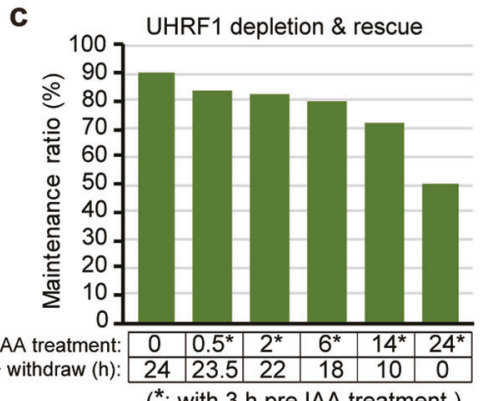

*: with $3 \mathrm{~h}$ pre IAA treatment.) d

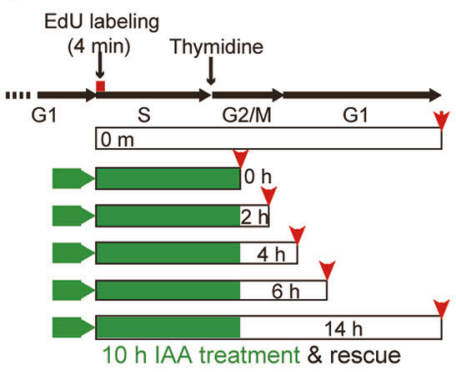

e

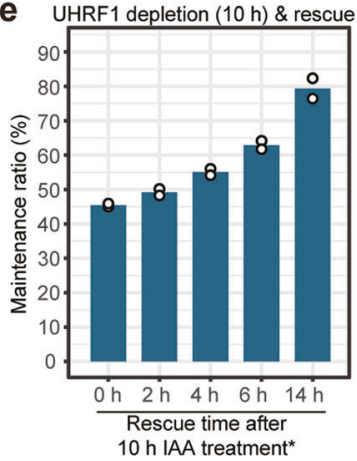

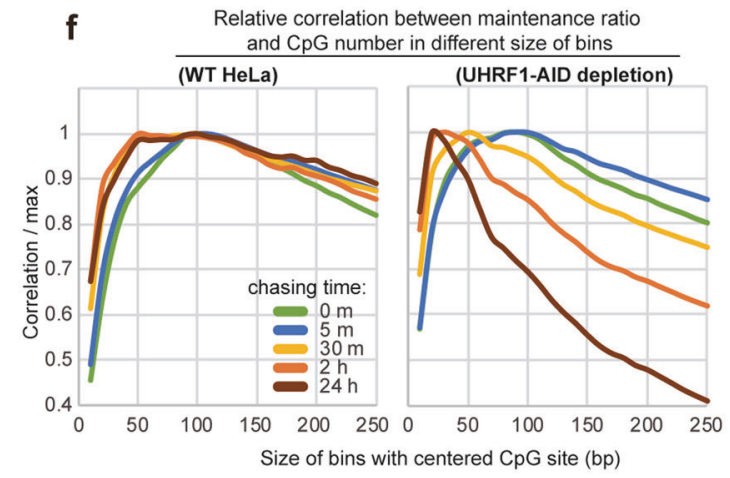

Fig. 4 The replication-uncoupled phase has potent maintenance capacity. a Maintenance kinetics of cells with depletion of the UHRF1 protein by IAA-induced degradation. WT cells are displayed for comparison. b Schematic diagram of UHRF1 depletion-rescue experiment set 1. The IAA treatment time and withdrawal time are as indicated. The green arrow represents a $3 \mathrm{~h} I A A$ treatment prior to cell cycle release. c Maintenance kinetics results of the experiment described in b. d Schematic diagram of UHRF1 depletion-rescue experiment set 2. The IAA treatment time and withdrawal time are as indicated. The green arrow represents a $3 \mathrm{~h}$ IAA treatment prior to cell cycle release. e Maintenance kinetics results of the experiment described in $\mathbf{d}$. Values from all replicates are indicated by circles. $\mathbf{f}$ Relative correlation between the maintenance ratio and CpG number in bins of different sizes. For each chase time point dataset, Pearson correlations were calculated for the covered CpG sites between the maintenance ratios and number of CpGs in bins containing the neighboring 10, 20, ..., 250 bp. For clarity of comparison, the correlation coefficients were normalized by the maximum value of the dataset for each time point.

UHRF1 protein still existed after IAA treatment (Supplementary information, Fig. S5a), and total depletion of UHRF1 would likely lead to even more severe impairment. Although UHRF1 depletion led to severe methylation maintenance failure, it had a minimal effect on cell cycle progression through one cell cycle (Supplementary information, Fig. S2d), indicating that cells do not have a methylation maintenance checkpoint system to guarantee the full restoration of methylation levels before entering the next cell cycle.

Then, we performed rescue experiments by withdrawing IAA and restoring UHRF1 production at various time points (Fig. 4b). Remarkably, even cells depleted of UHRF1 for $14 \mathrm{~h}$ could attain a maintenance ratio exceeding $72 \%$ when a rescue period of $10 \mathrm{~h}$ was provided (Fig. 4C), indicating that replication-uncoupled maintenance occurred robustly at the post-S phase stages. We also performed another set of rescue experiments in which UHRF1 was depleted throughout the entire $S$ phase ${ }^{64}$ and Hammer-seq was performed at various time points after UHRF1 restoration (Fig. 4d). Clearly, the maintenance ratio recovered steadily after UHRF1 restoration (Fig. 4e). These results collectively support the hypothesis that cells have a highly potent post-replication maintenance backup capacity that can correct epigenetic information loss due to disruption of the replication-coupled maintenance system.

As previously mentioned, the number of neighboring $\mathrm{CpGs}$ positively correlates with the methylation maintenance ratio. To evaluate the effective range of neighboring $\mathrm{CpGs}$, we compared the correlation between the number of neighboring CpGs and the maintenance ratio for $\mathrm{CpG}$-centered bins of different sizes. The correlation peaks (see the Materials and methods section for details) at the late time points ( 2 and $24 \mathrm{~h}$ ) displayed a clear leftward shift compared with those at the early time points $(0 \mathrm{~min}$, $5 \mathrm{~min}$, and $30 \mathrm{~min}$ ) from $100 \mathrm{bp}$ to $50 \mathrm{bp}$ (Fig. 4f, left panel). These results suggest that the influence range of neighboring $\mathrm{CpGs}$ is narrower during replication-uncoupled maintenance. To confirm this hypothesis, we performed a similar analysis in UHRF1depleted cells, in which methylation maintenance is achieved primarily through replication-uncoupled maintenance. Consistent with the above findings, the correlation peaks at very early time points ( 0 and $5 \mathrm{~min}$ ) were still at $100 \mathrm{bp}$, but the leftward shift became more obvious with time, and a sharp peak appeared at $20 \mathrm{bp}$ at the $24 \mathrm{~h}$ chase time point (Fig. $4 \mathrm{f}$, right panel). This finding again supports the idea that the influence range of neighboring $\mathrm{CpGs}$ is wider during replication-coupled maintenance than during replication-uncoupled maintenance.

Nucleosome occupancy and H3K9 methylation specifically regulate replication-uncoupled maintenance

Although replication-uncoupled maintenance displayed a remarkably high capacity, its kinetics were obviously slower than those of replication-coupled maintenance (Fig. 1b). Several reasons could contribute to this difference, including the aforementioned interactions between the replication machinery and maintenance machinery, such as the interactions between PCNA-DNMT1 and LIG1-UHRF1, which potentiates replication-coupled maintenance. In addition, replication-uncoupled maintenance operates on a more challenging substrate - chromatinized DNA. Although nucleosomes offer multiple mechanisms to interact with and even stimulate the maintenance machinery, ${ }^{20}$ chromatin likely acts as a barrier to DNA methylation. 


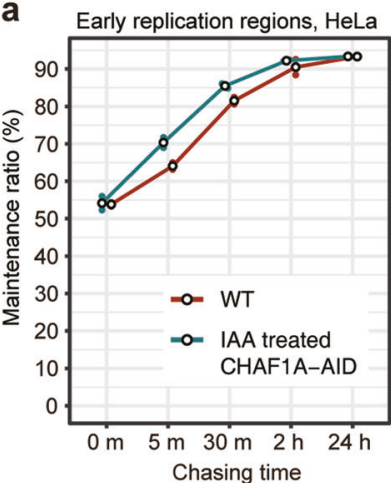

b Early replication regions, HeLa

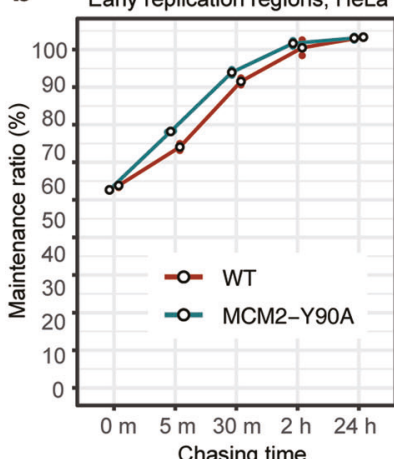

d

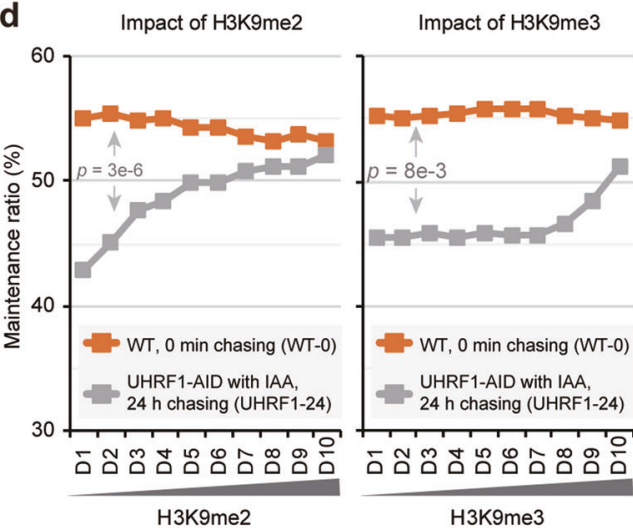

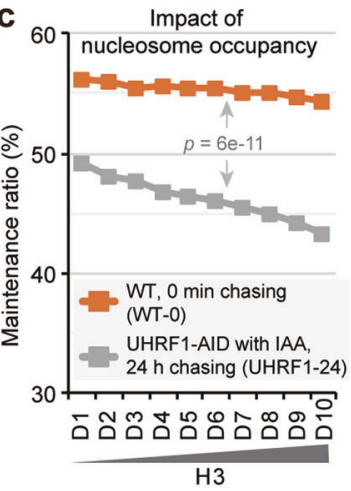
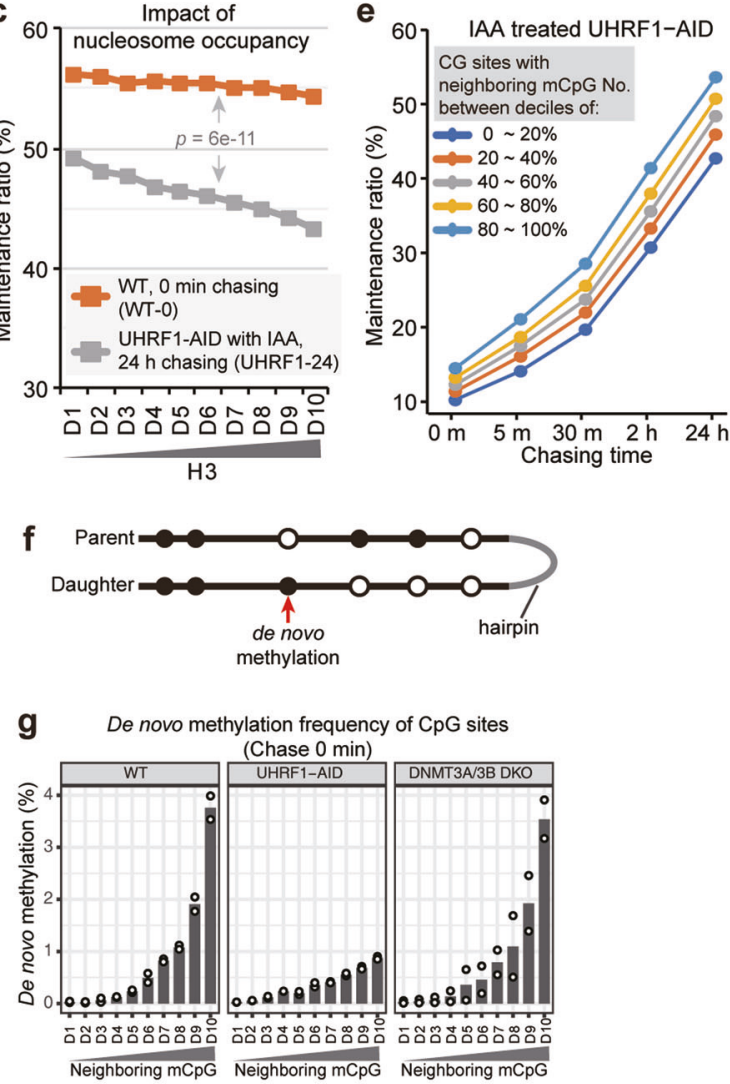

Fig. 5 Nucleosome occupancy decelerates maintenance methylation kinetics in the replication-uncoupled phase. a Maintenance kinetics of IAA-treated CHAF1A-AID cells. b Maintenance kinetics of MCM2-Y90A cells. Symbols in a, b are used similarly to those in Fig. 2b. c Maintenance kinetics across CpG groups with different nucleosome occupancies under the WT-0 and UHRF1-24 conditions. d Maintenance kinetics across CpG groups with different H3K9me2 levels (left panel) and H3K9me3 levels (right panel) under the WT-0 and UHRF1-24 conditions. e Maintenance kinetics across CpG groups with different ranges of neighboring mCpGs in UHRF1-depleted cells. CpGs are grouped by every two deciles of neighboring $\mathrm{mCpGs}$. $\mathbf{f}$ Schematic of the definition of de novo methylation events detected by Hammer-seq. g De novo methylation frequencies of CpG sites with different neighboring mCpG densities in WT cells, UHRF1-depleted cells and DNMT3A/ 3B DKO cells. Values from all replicates are indicated by circles.

To test this idea, we generated a mutant cell line with homozygous knock-in of CHAF1A-AID (3 alleles in HeLa cells) (Supplementary information, Fig. S6a). Inducing the degradation of CHAF1A, a key component of the chromatin assembly complex $\mathrm{CAF} 1,{ }^{65}$ resulted in faster replication-uncoupled maintenance, although replication-coupled maintenance was largely normal (Fig. 5a). To further confirm the effect of nucleosome occupancy on the regulation of DNA methylation maintenance, we generated a homozygous MCM2-Y90A knock-in (3 alleles in HeLa cells) cell line (Supplementary information, Fig. S6b). The MCM2-Y90A mutation disrupts the interaction between MCM2 and histone $\mathrm{H} 3-\mathrm{H} 4$, which reduces parental nucleosome recycling during replication. ${ }^{47,66,67}$ Interestingly, a similar trend of a faster replication-uncoupled maintenance rate was observed in MCM2-Y90A cells (Fig. 5b).

The above data suggest that nucleosome occupancy has a greater influence on replication-uncoupled maintenance than on replication-coupled maintenance. To more specifically investigate the mechanisms governing replication-coupled and replicationuncoupled maintenance, we selected two conditions for further analysis: WT cells with a 0 min chase (WT-0) and IAA-treated UHRF1-AID cells with a $24 \mathrm{~h}$ chase (UHRF1-24). We selected these two conditions for comparison for two reasons: 1) they displayed comparable maintenance ratios (Fig. 4a); 2) the WT-0 condition mostly reflected replication-coupled maintenance, whereas the UHRF1-24 condition mostly reflected replication-uncoupled maintenance. Obviously, nucleosome occupancy negatively impacted replication-uncoupled maintenance but had a much lower effect on replication-coupled maintenance (Fig. 5c).

We also used the WT- 0 and UHRF1-24 conditions to analyze the impact of $\mathrm{H} 3 \mathrm{~K} 9$ methylation on the regulation of replicationcoupled and replication-uncoupled maintenance. Both H3K9me2 and $\mathrm{H} 3 \mathrm{~K} 9 \mathrm{me} 3$ displayed little impact on replication-coupled maintenance, but H3K9 methylation-enriched regions were clearly favored for replication-uncoupled maintenance (Fig. 5d) when limited UHRF1 was available. These results reiterated our earlier observations that H3K9 methylation did not affect replicationcoupled maintenance but did affect replication-uncoupled maintenance (Fig. 3a).

To answer whether the density of neighboring $\mathrm{mCpGs}$ also plays a positive role during replication-uncoupled stage, we analyzed the UHRF1-AID dataset in which the majority of methylation maintenance occurred in replication-uncoupled phase due to a shortage of UHRF1 proteins, and we found a positive impact of neighboring $\mathrm{mCpG}$ density in facilitating replication-uncoupled maintenance (Fig. 5e).

Back to the distinct maintenance kinetics between the leading and lagging strands (Fig. $1 \mathrm{~h}-\mathrm{j}$ ), we asked whether the LIG1-UHRF1 interaction may cause maintenance kinetics to favor the lagging strand and whether an MCM2-mediated nucleosome deposition bias between the leading and lagging strands ${ }^{47,67}$ may contribute to the distinct maintenance kinetics between the leading and lagging strands. Therefore, we analyzed the maintenance kinetics of the leading and lagging strands in the LIG1-K126A mutant cell 
line, MCM2-Y90A mutant cell line and all other mutant cell lines used in this study. In all tested conditions, lagging strand maintenance remained faster than leading strand maintenance (Supplementary information, Fig. S7). Although we could not rule out minor contributions from these perturbed mechanisms, we concluded that there are other mechanism(s) that play a more profound role in regulating the differential maintenance kinetics between the lagging and leading strands. Based on our observations that nucleosome occupancy affects maintenance kinetics (Fig. $5 a-c$ ), we speculated that nucleosome assembly likely occurs slightly more rapid at the leading strand than at the lagging strand, allowing a slightly longer nucleosome-free period and faster DNA methylation maintenance at the lagging strand.

Occurrence of de novo methylation mediated by DNMT1 during methylation maintenance

Hammer-seq simultaneously measures the methylation status of the same CpG sites in the parent and daughter strands, allowing effective detection of de novo methylation at a single-base resolution. We identified CPG sites that were unmethylated in the parent strand but became methylated in the daughter strand and defined the associated events as de novo methylation events (Fig. 5f). Similar to the findings for maintenance methylation, CpG sites with more neighboring $\mathrm{mCpG}$ sites displayed higher de novo methylation frequencies, with approximately $3.8 \%$ de novo methylation in the highest group at the 0 min chase time (Fig. $5 \mathrm{~g}$ ). Due to its replication-coupled occurrence and its dependency on UHRF1 (Fig. 5g), this de novo methylation was likely catalyzed by DNMT1. HeLa cells express low levels of DNMT3A and DNMT3B; nevertheless, to confirm our hypothesis, we generated cell lines with double homozygous knockout of DNMT3A ( 3 alleles in HeLa cells) and DNMT3B (4 alleles in HeLa cells) (Fig. 2a; Supplementary information, Figs. S2c and S6c) and found that de novo methylation remained at comparable levels (Fig. $5 \mathrm{~g}$ ). These data provide a quantitative assessment of de novo methylation mediated by DNMT1 at a single-base resolution in vivo and support a role of DNMT1mediated de novo methylation in maintaining methylome homeostasis.

Imperfect methylation inheritance contributes to deterioration of the methylome during aging and tumorigenesis

Despite of having a system with a robust replication-coupled maintenance (Fig. 1b) and a very strong replication-uncoupled maintenance backup (Fig. 4c, e), mitotic inheritance of DNA methylation is unlikely to be perfect. And it is questionable whether subtle imperfections associated with such a system may accumulate over time. Among all examined features, the neighboring $\mathrm{mCpG}$ density displayed a very strong positive correlation with both the maintenance rate and de novo methylation frequency in HeLa cells (Figs. 1e, $5 g$ ). This finding prompted us to ask whether regions with a lower CpG density are more vulnerable to methylation loss during mitotic division. Since we generated the UHRF1-AID cell line, and it displayed reduced UHRF1 expression (Supplementary information, Fig. S5a, lane 2 vs lane 1) and methylation levels (Fig. 2a), we used the parent strand methylation level as the bulk methylation level and compared the difference between WT and UHRF1-AID cells at regions with different $\mathrm{CpG}$ densities. Clearly, the difference between UHRF1AID cells and WT cells was greater at regions with fewer CpG sites (Fig. 6a), supporting the hypothesis that $\mathrm{CpG}$ contexts with slower maintenance kinetics and low de novo methylation frequency are prone to accumulating more methylation defects during mitotic division.

Cancer cells and mitotic cells from aged tissue samples experience extensive mitotic division, and both of them are generally hypomethylated. ${ }^{39,43}$ Recently, it was reported that in tumor samples and aged tissue samples, DNA methylation loss preferentially occurs in CpG sites in the solo-WCGW context, and this selective hypomethylation serves as a clock to indicate the history of mitotic division. ${ }^{40}$ Whether this characteristic hypomethylation is caused by specific failure of methylation maintenance or preferential active demethylation remains unknown. Interestingly, CpG sites in the solo-WCGW context displayed the slowest maintenance kinetics (Fig. $6 \mathrm{~b}$ and Supplementary information, Fig. S6d) and the lowest de novo methylation frequencies (Fig. 6c). Therefore, the hypomethylation signature observed in aged tissue samples and tumor cells that underwent extensive mitotic division was likely caused by the failure of methylation maintenance.

Recent progress has demonstrated that DNA methylation is a valuable biomarker for age prediction. ${ }^{34-36}$ In an extensive human cohort study, the methylome of peripheral blood leukocytes from 421 Swedish persons ranging in age from 14 to 94 were examined via a HumanMethylation 450 BeadChip, and among the 476 k CpGs covered in the study, $18 \%$ exhibited aging-associated methylation loss. ${ }^{68}$ We analyzed the associated data and categorized these CpGs into three groups (increased, stable, and decreased during aging; see the Materials and methods section for details). We determined the maintenance kinetics for the above three groups and found that $\mathrm{CpGs}$ with methylation loss during aging clearly displayed the slowest maintenance kinetics and that the other two groups had similar maintenance kinetics (Fig. 6d). Moreover, CpGs with methylation loss during aging contained a 22-fold higher frequency of solo-WCGW sites than CpGs acquiring methylation during aging (Fig. 6e).

Collectively, our results with independent aging datasets suggest that imperfect maintenance during mitotic division is an important cause of aging-associated specific methylation loss.

LSH facilitates replication-uncoupled maintenance in late Sreplicating regions

Methylation loss in aging and tumorigenesis occurs both in early and late $S$ replication domains, and domain-wide methylation loss occurs primarily in late $S$ domains. ${ }^{40,69-71}$ We measured the maintenance kinetics in late $S$ replication domains with Hammerseq and compared that with early $S$ domains (Fig. 7a and Supplementary information, Fig. S8a). Interestingly, the overall replication-coupled maintenance ratio of late $\mathrm{S}$ domains was even higher than that of early $\mathrm{S}$ domains, which was $70 \%$ at the 0 min chase time point (Fig. 7a). However, the maintenance ratio of late $\mathrm{S}$ domains was surpassed by that of early $\mathrm{S}$ domains after $30 \mathrm{~min}$ (Fig. 7a). Importantly, the positive role of the number of neighboring $\mathrm{mCpGs}$ in facilitating methylation maintenance remained in late $S$ replication domains (Supplementary information, Fig. S8b, c).

The late $S$ replication domains displayed significantly slower replication-uncoupled maintenance kinetics than early $S$ replication domains (Fig. 7a). Several events likely contribute to this pattern: 1) late $S$ replication domains are enriched in heterochromatin regions with higher nucleosome occupancy, which negatively regulates replication-uncoupled methylation maintenance (Fig. $5 \mathrm{a}-\mathrm{C}$ ); and 2 ) late $\mathrm{S}$ domains are AT-rich regions with $\mathrm{a}$ lower CpG density, ${ }^{72}$ which is also a disadvantage for maintenance (Fig. 1e; Supplementary information, Figs. S1f and S8b, c).

Chromatin remodelers could help to overcome the barrier effect of nucleosome occupancy. Notably, LSH, a chromatin remodeler, is essential for global methylation levels. ${ }^{73}$ We wondered whether and how LSH regulates methylation maintenance. We generated a homozygous LSH-AID knock-in cell line ( 3 alleles in HeLa cells) (Supplementary information, Fig. S8d) and performed Hammer-seq profiling for early $S$ and late $S$ replication domains in IAA-treated LSH-AID cells (Supplementary information, Fig. S8e). Although LSH depletion had a minimal effect on the maintenance kinetics of early $S$ replication domains (Fig. $7 \mathrm{~b}$ ), it caused an apparent delay of replication-uncoupled 
a

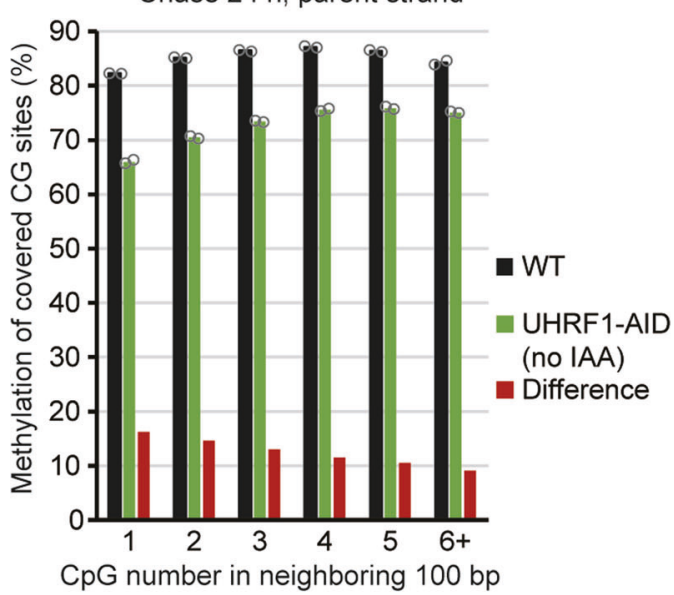

b

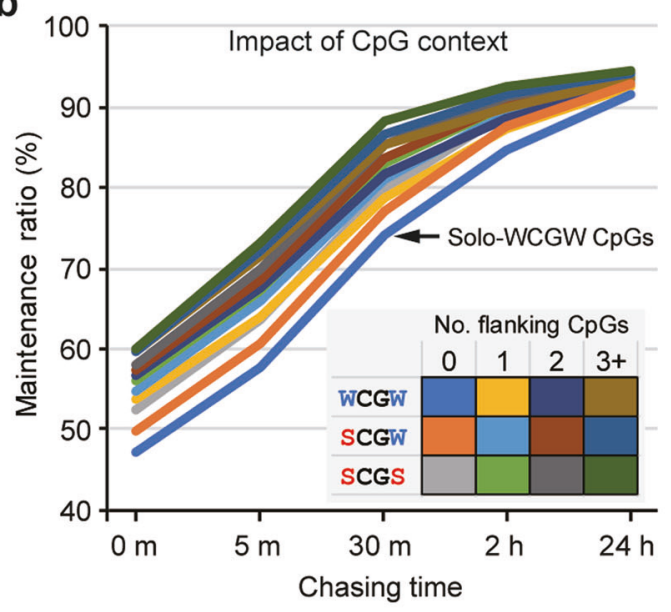

C

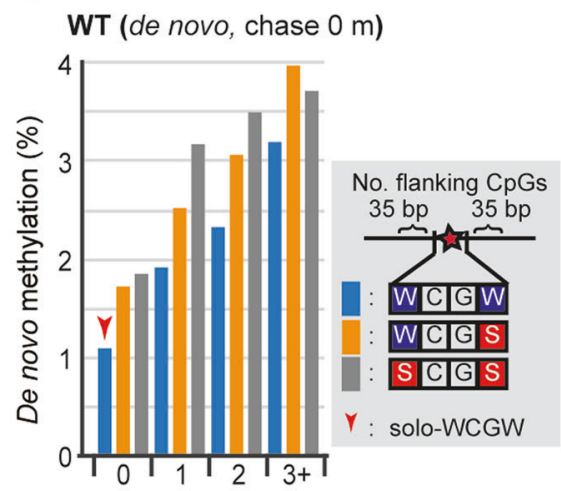

No. of flanking $\mathrm{CpGs}$

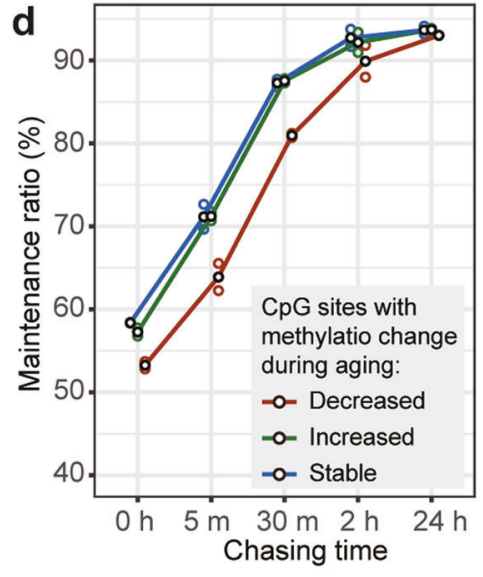

e

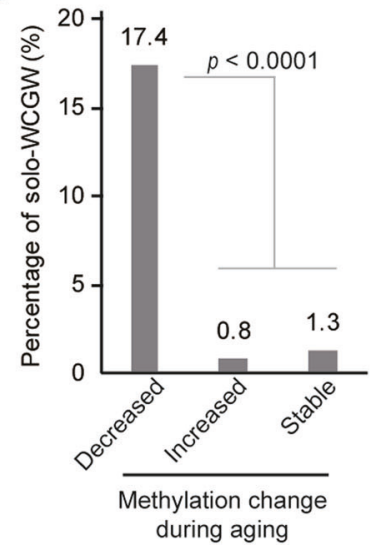

Fig. 6 Solo-WCGW CpGs and other CpGs with preferential loss of methylation during aging display a low maintenance efficiency. a Differences in methylation levels between WT and UHRF1-AID cells (no IAA treatment) across CpG groups with different numbers of neighboring CpGs. Parent strands at the $24 \mathrm{~h}$ chase time point were used to determine the methylation level in UHRF1-AID and WT cells. Values from all replicates are indicated by circles in $\mathbf{a}$ and $\mathbf{d}$. $\mathbf{b}$ Comparison of the maintenance kinetics of CpGs within different local sequence contexts. c Comparison of the de novo methylation frequency of CpGs within different local sequence contexts at the 0 min chase time point. d Maintenance kinetics of CpG groups that experience differential methylation changes during aging. See the Materials and methods section for the definitions of increased, decreased and stable groups of CpG sites. e Percentage of CpGs in the solo-WCGW context in CpG groups that experience differential methylation changes during aging.

maintenance in late $S$ replication domains (Fig. 7c). These data suggest that in addition to the neighboring $\mathrm{mCpG}$ density, chromatin remodeling is also highly important for methylation maintenance, especially for replication-uncoupled maintenance in heterochromatin regions.

Mouse ES cells exhibit similar maintenance kinetics to those in HeLa cells

Finally, to confirm our findings in a different cell type, we performed Hammer-seq in mouse ES cells, which have a quite different methylome pattern (Supplementary information, Fig. S8f) compared with HeLa cells, without cell cycle synchronization. We pulsed mouse ES cells with EdU for 4 min and chased for 0 min and $2 \mathrm{~h}$. The parent strand methylation levels were stable at these time points, but the daughter strand methylation levels and maintenance ratios increased after two hours (Fig. 7d). These results supported the existence of replication-uncoupled maintenance in mouse ES cells. Moreover, at the $0 \mathrm{~min}$ chase time point, mouse ES cells also exhibited lower maintenance ratios in regions with a low CpG density (Fig. 7e), and CpGs in the soloWCGW context displayed the lowest maintenance ratios (Fig. 7f).
These results confirm that the main conclusions drawn in HeLa cells also apply to mouse ES cells.

\section{DISCUSSION}

Hammer-seq allows a more quantitative measurement of maintenance kinetics

The Hammer-seq technique developed in this study allows simultaneous measurement of the methylation status of the parent and daughter strands at the single-molecule level. This approach offers two important advantages. First, Hammer-seq eliminates any potential averaging effect generated in the bulk measurements, thus providing more precise and accurate quantification. With this improvement, the Hammer-seq results not only support the observation of a replication-uncoupled phase, ${ }^{11}$ but also provide opportunities to reveal locus-specific maintenance kinetics (Fig. 1c, d), to dissect the roles of H3K9 methylation and nucleosome occupancy in the regulation of methylation maintenance (Figs. 3a, 5a-d) and to reveal the subtle differences between leading and lagging strand maintenance kinetics (Fig. $1 \mathrm{~h}-\mathrm{j}$ ). Second, Hammer-seq provides a tool to study 

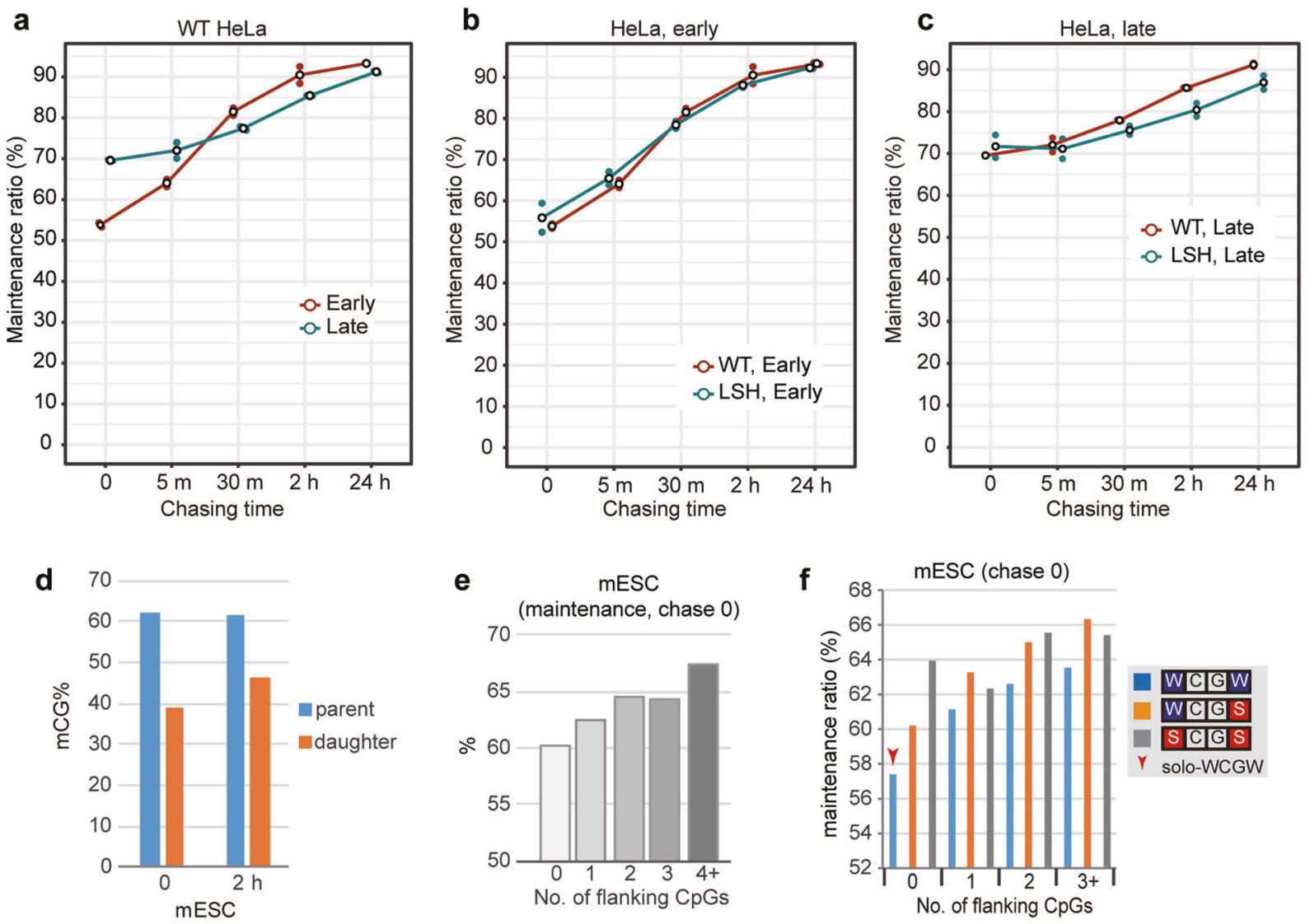

Fig. 7 The replication-uncoupled phase in late $\mathbf{S}$ replication domains shows a decreased maintenance rate and is highly dependent on LSH. a Maintenance kinetics of late $S$ domains. For labeling of late $S$ domains, EdU was added at $6 \mathrm{~h}$ after entry into $\mathrm{S}$ phase. Early $\mathrm{S}$ domains are shown for comparison. Symbols in a-c are used similarly to those in Fig. 2 b. b Maintenance kinetics of LSH-AID early S domains. WT early $S$ domains are shown for comparison. c Maintenance kinetics of LSH-AID late S domains. For LSH-AID cells, IAA was added $3 \mathrm{~h}$ after entry into $\mathrm{S}$ phase. WT late $\mathrm{S}$ domains are shown for comparison. $\mathbf{d}$ Overall methylation levels in parent strands and daughter strands at the 0 min and $2 \mathrm{~h}$ chase time points in mESCs. e Maintenance ratio across CpG groups with different numbers of neighboring CpGs at the 0 min chase time point in $\mathrm{mESCs}$. $\mathbf{f}$ Maintenance ratio of CpGs in different local sequence contexts at the 0 min chase time point in mESCs.

de novo methylation at a single-base resolution (Fig. $5 f, g$ ), which was not possible before.

A full picture revealing mechanisms regulating methylation maintenance

In addition to showing the sensitive and quantitative properties of Hammer-seq, by combining this technique with studies in various mutant cell lines with perturbations in different mechanisms regulating or potentially regulating methylation maintenance, this study organizes individual mechanisms into a global picture (Supplementary information, Fig. S8g) and reveals their distinct roles in different stages (replication-coupled and -uncoupled phases) and in different regions (early $S$ and late $S$ replicating regions, etc.).

In addition, combining Hammer-seq with additional studies in mutants provided insights regarding ambiguous aspects of methylation maintenance regulation. For example, nucleosomes play a central part in UHRF1 and DNMT1 chromatin association and activation, but it can also prevent the access of substrate DNA from the DNA methylation maintenance machinery. Here, the differential contributions of nucleosome occupancy in the replication-coupled and replication-uncoupled phases (Fig. 5c) and the accelerated replication-uncoupled maintenance kinetics in CHAF1A-depleted cells and MCM2-Y90A cells (Fig. 5a, b) reveal a role for chromatin as a barrier to methylation maintenance, especially in the replication-uncoupled phase. These effects are probably the major reason that UHRF1 and DNMT1 have evolved multiple mechanisms involved in histone recognition, histone ubiquitination, and ubiquitination-dependent activation.

In addition to H3K9 methylation and nucleosome occupancy, which predominantly affect replication-uncoupled maintenance, methylated CpGs strongly potentiate the methylation maintenance of neighboring CpGs (Figs. 1e, 5e and Supplementary information, Fig. S8b), consistent with the observation that methylated CpGs tend to be distributed together. ${ }^{74}$

The capacity and necessity of replication-uncoupled maintenance The passage of the replication fork is a very fast event, ${ }^{75}$ and replication-coupled nucleosome assembly occurs immediately after the passage of the replication fork. ${ }^{76}$ Given the inhibitory role of nucleosome occupancy in methylation maintenance (Fig. $5 a-c$ ), only a short time window is available for replication-coupled maintenance. Therefore, although replication-coupled maintenance is fairly effective, it cannot fully restore the methylome. Thus, replication-uncoupled maintenance is absolutely required for preventing the loss of epigenetic information.

Importantly, replication-uncoupled phase reserves a surprisingly strong maintenance capacity (Fig. 4c, e). Although only a small portion of methylation is maintained at replication-uncoupled phase in normal condition (Fig. 1b), when replication-coupled maintenance is greatly impaired, the majority of the methylome can be maintained in the replication-uncoupled phase (Fig. 4c, e). The backup effect of replication-uncoupled maintenance provides 
a strong buffering effect on methylation inheritance, which is important for maintaining cell identity.

With such a powerful replication-uncoupled maintenance system as a backup, an intriguing question is why the methylation maintenance machinery has evolved multiple mechanisms to associate with the replication machinery, such as the DNMT1PCNA and UHRF1-LIG1 interactions. A reasonable interpretation is that replication-uncoupled maintenance is affected by different mechanisms, such as H3K9 methylation and nucleosome occupancy. The interactions between the replication machinery and methylation maintenance machinery help to accelerate replication-coupled maintenance (Fig. 2b, c), which not only helps to reduce the workload of replication-uncoupled maintenance but also likely reduces the potential methylome skew that might occur during replication-uncoupled maintenance.

The role of chromatin structure in methylation maintenance Nucleosome occupancy clearly negatively impacts replicationuncoupled maintenance (Fig. $5 a-c$ ). This finding suggests that chromatin structure may also affect methylation maintenance. Indeed, LSH, a chromatin remodeler, preferentially regulates methylation maintenance in late $S$ replication domains (Fig. $7 b$, c). This observation is highly interesting, because late $\mathrm{S}$ replication domains are enriched in heterochromatin, which is known to experience preferential methylation loss in cancer cells and aged tissue samples.

Imperfect methylation maintenance is an important contributor to aging- and tumorigenesis-associated methylation loss

Aging- and tumorigenesis-associated methylation loss has been observed repeatedly, but the underlying mechanisms are far from clear. ${ }^{37-39}$ Altered expression of methylation-related enzymes such as DNMTs ${ }^{77}$ and TETs $s^{78,79}$ or altered levels of coenzymes such as SAM or a-ketoglutarate ${ }^{80}$ were observed in aged cells or tissues, which could offer potential explanations. However, methylation loss of late $S$ replication domains start at fetal development stage, ${ }^{40}$ and aging-associated DNA methylation changes occur at the very beginning of birth. ${ }^{81}$ Thus, those potential mechanisms occurring at later stages do not explain the observation that aging-associated methylation change starts from the very beginning of life.

In addition to these potential mechanisms, our findings about the intrinsic imperfection of methylation maintenance predict that even when all components of the methylation/demethylation machineries and coenzymes are normal, selective methylation loss is an inevitable consequence of extensive mitotic division, which can be easily observed during aging and tumorigenesis.

The imperfect characteristic of the methylation maintenance system is supported by the relationship between the neighboring $\mathrm{mCpG}$ density and maintenance efficiency: low neighboring $\mathrm{mCpG}$ density is a disadvantage for methylation, with slower maintenance kinetics and lower de novo methylation frequencies in this context (Figs. 1e, $5 \mathrm{~g}$ and Supplementary information, Fig. S8b). These regions tend to exhibit methylation loss and reduced $\mathrm{mCpG}$ densities, which in turn further increases the vulnerability to methylation loss through further mitotic divisions during aging and tumorigenesis because of the negative effect of low neighboring $\mathrm{mCpG}$ density on methylation maintenance.

Interestingly, unlike HeLa cells and most immortal somatic cells, ES cells did not show obvious methylation loss after extensive mitotic cell division (Supplementary information, Fig. S8f). The presence of distinct methylation regulators in ES cells likely provides a possible explanation. In ES cells, the highly dynamic equilibrium between active demethylation by TET enzymes and de novo methylation by DNMT3s likely reduces the epigenetic perturbation caused by cell division; whereas most somatic cells rely on the DNMT1 and UHRF1 to maintain DNA methylation. ${ }^{82}$
Methylation maintenance failure during aging and tumorigenesis: cause or consequence?

Our study found that the imperfection of methylation maintenance mediated by DNMT1 during mitotic division is an important contributor to the deterioration of the methylome during aging and tumorigenesis. Obviously, an important question is whether methylation maintenance failure is simply a consequence of extensive cell division during aging and tumorigenesis or whether it may also contribute to aging and tumorigenesis. Undoubtedly, methylation maintenance imperfection is an intrinsic property of mitosis, and the accumulation of maintenance failure is an inevitable consequence of extensive cell division, which is associated with aging and tumorigenesis.

Methylation loss due to imperfect mitotic inheritance is not likely to contribute to early events in aging and tumorigenesis because of the stochastic nature of methylation loss in each cell cycle and the requirement for a sufficient number of cell divisions to accumulate a significant change.

However, both aging and tumorigenesis are long-term events that allow methylome deterioration to accumulate. Thus, we strongly believe that such a failure of methylation maintenance likely becomes physiologically relevant after enough cell divisions. Indeed, an acceleration of DNA methylation changes in aging was reported to be associated with increased occurrence of agerelated diseases. ${ }^{83}$ Moreover, many aberrant DNA methylation changes in cancer also occur during aging, and it was proposed that DNA methylation changes in aging might contribute to tumorigenesis. ${ }^{39}$ Specifically, LINE1 demethylation and derepression occur in aging, ${ }^{33}$ activating the interferon pathway and promoting age-associated inflammation. ${ }^{84}$ Interestingly, we found repetitive elements such as LINEs show slow maintenance kinetics (Fig. 1c), which suggests that the imperfection of DNA methylation maintenance contribute to methylation loss of those repetitive elements during aging.

In summary, we established a method dedicated to the precise measurement of methylation maintenance and pinpointed mechanisms regulating replication-coupled and replicationuncoupled maintenance. These findings not only provide a global picture of methylation maintenance kinetics and relevant mechanisms but also raise an important claim that the intrinsic imperfection of methylation maintenance contributes to the inevitable deterioration of the methylome during mitotic division and likely contributes to aging and tumorigenesis. Our findings also predict that the selective methylation loss occurring in aging is irreversible.

\section{MATERIALS AND METHODS}

Cell lines and cell culture

Human HeLa S3 cells were obtained from ATCC, and WT cells and mutant cells derived from HeLa S3 were maintained under standard in vitro conditions $\left(37^{\circ} \mathrm{C}, 5 \% \mathrm{CO}_{2}\right)$ in DMEM medium (Hyclone, $\mathrm{SH} 30022)$ supplemented with $10 \%$ fetal bovine serum (FBS) (BI, 04010-1ACS), 1\% Penicillin-Streptomycin (Gibco, 15140122).

Mouse embryonic stem cells were obtained from ATCC, and cultured on gelatin coated dishes in ES culture medium containing DMEM (ThermoFisher 11995), supplemented with 15\% FBS (Hyclone, 30070.03), 1\% non-essential amino acids (Merck, M7145), 1\% L-glutamine (BBI Life Sciences, E607004), 0.1\% $\beta$-mercaptoethanol (Gibco, 21985023), $0.01 \%$ LIF $\left(10^{7} \mathrm{U} / \mathrm{mL}\right)$, and $1 \%$ PenicillinStreptomycin (Gibco, 15140122).

\section{Hammer-seq}

HeLa cells were synchronized at G1/S entrance with thymidine/ aphidicolin double block. Briefly, after culturing in the presence of $2 \mathrm{mM}$ thymidine (Sigma, T1895) for $18 \mathrm{~h}$, the cells were cultured for another $9 \mathrm{~h}$ without thymidine and then were further cultured in the presence of $1 \mu \mathrm{g} / \mathrm{mL}$ aphidicolin (Abcam, 38966-21-1) for 
another $14 \mathrm{~h}$. Aphidicolin were removed and then the cells were pulse labeled with $20 \mu \mathrm{M}$ EdU (Thermo Fisher, E10187) for 4 min. Cells were chase for different time length ( 0 min to $24 \mathrm{~h}$ ) after the removement of EdU. Harvest cells and extract DNA. DNA fragmentation to around $150 \mathrm{bp}$ was performed by sonication (Covaris M220, following the manufacturer's instructions). Sizeselection of DNA fragments ranging from $100 \mathrm{bp}$ to $200 \mathrm{bp}$ was performed with AMPure beads $(1.05 \times$ to $2.8 \times)$ (Beckman, A63881). Biotin was conjugated to EdU-labeled DNA by Click-it reaction in the buffer containing Tris, pH $7.515 \mathrm{mM}$; Biotin azide (Sigma, 762024) 400 uM; $\mathrm{CuSO}_{4}$ (Sigma, 209198)/THPTA (Sigma, 762342) mix (1:5) (100 $\mu \mathrm{M}: 500 \mu \mathrm{M})$; freshly prepared sodium ascorbate (Sigma, A4034) $5 \mathrm{mM}$ with incubation at $34^{\circ} \mathrm{C}$ for 20 min. DNA was purified with MinElute PCR purification kit (Qiagen, 28004). The click-it reaction system is modified from the previous reports. $^{85,86}$ End repair (NEB, E6050), dA-tailing (NEB, E6053) and adapter ligation (NEB, E6056) were carried out following the manufacturer's instructions. Hairpin adapter and methylated sequencing adapter were pre-mixed at the molar ratio of 3:1 before adapter ligation. After ligation, 1.4× AMPure beads were added to remove un-ligated adapters. Adapter ligatedDNA was dissolved in $1 \times$ TE buffer then was incubated with M280 streptavidin beads (Thermo Fisher, 11205D) in binding/ washing buffer: $5 \mathrm{mM}$ Tris- $\mathrm{Cl}$, pH 7.5, $1 \mathrm{M} \mathrm{NaCl}, 0.5 \mathrm{mM}$ EDTA, pH 8.0 for $20 \mathrm{~min}$. After rinsing in binding/washing buffer containing $0.5 \%$ IGEPAL CA-630 (Sigma, I3021) for 6 times, the beads was

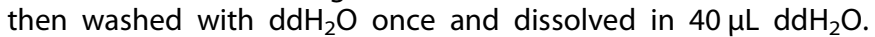
DNA on beads was subjected to bisulfite conversion (Qiagen, 59104) and then bisulfite-converted DNA was amplified in PCR (KAPA KR0413) and was separated in $2 \%$ agarose gel. The larger band ranging from $300 \mathrm{bp}$ to $500 \mathrm{bp}$ was hairpin-ligated sample. Band was recovered with gel-extraction kit (TIANGEN, DP208) and cleaned up with AMPure beads $(0.9 \times)$. The resulting DNA was dissolved in $0.1 \times$ TE buffer. The Hammer-seq library is ready for Next Generation Sequencing.

\section{Nascent EdU-IP-seq}

This method is used to immuno precipitate nascent strand DNA and determine its methylation state. Cell synchronization, pulsechase, cell-harvest and DNA extraction were performed as described in the previous parts. DNA fragmentation around $300 \mathrm{bp}$ was performed by sonication (Covaris M220, following the manufacturer's instructions). Size-selection of DNA fragments ranging from $200 \mathrm{bp}$ to $400 \mathrm{bp}$ was performed with AMPure beads $(0.6 \times$ to $1.0 \times$ ) (Beckman, A63881). Click-it reaction is the same as described in Hammer-seq. End repair (NEB, E6050), dAtailing (NEB, E6053) and adapter ligation (NEB, E6056) were carried out following the manufacturer's instructions. In adapter ligation step, only methylated sequencing adapter was used. After ligation, AMPure beads $(1 \times)$ were added to remove unligated adapters. Incubate DNA at $100^{\circ} \mathrm{C}$ for $10 \mathrm{~min}$, and put on ice immediately after that. Incubate on ice for more than $3 \mathrm{~min}$. Incubate DNA with M280 streptavidin beads in binding/washing buffer at $4{ }^{\circ} \mathrm{C}$ for $20 \mathrm{~min}$ and then wash with binding/washing buffer containing $0.5 \%$ IGEPAL CA-630 at $4{ }^{\circ} \mathrm{C}$ twice followed by wash at room temperature for 3 more times. Wash with $\mathrm{dd}_{2} \mathrm{O}$ once and dissolve beads in $40 \mu \mathrm{L} \mathrm{ddH}_{2} \mathrm{O}$. Bisulfite conversion with DNA on beads and PCR amplification were the same as described in Hammer-seq. Clean up with AMPure beads $(1 \times)$. The Nascent EdU-IP-seq library is ready for Next Generation Sequencing.

Generation of knock-in (KI) and knock-out (KO) cell lines

1. Plasmid construction

1) Guide RNA (gRNA) design and clone

All the gRNAs used in this work were cloned to lentiCRISPR v2 plasmid (Addgene plasmid \#52961), which can express gRNA, SpCas9, and a puromycin resistant gene in a single plasmid. ${ }^{87}$
gRNA design and evaluation were based on the information from websites http://crispor.tefor.net/crispor.py and http://crispr. dfci.harvard.edu/SSC/ ${ }^{88,89}$.

2) Construction of plasmid-based donor, which was used as repair template for DNMT1 H170V KI (DNMT1-H170V) and UHRF1 Y188V/Y191V KI (UHRF1-TTD) cell lines.

Homology arms were ligated to a plasmid as the repair template in those two $\mathrm{KI}$ cell line construction. Homology arms flanking the site of alteration are about $1 \mathrm{~kb}$ in length. Firstly, a 2 $\mathrm{kb}$ fragment centered the alteration site was amplified and ligated to pEASY-Blunt Zero plasmid (TransGen Biotech, CB501). Site directed mutagenesis by PCR was used to introduce desired codon mutation. The gRNA PAM site was mutated to prevent SpCas9 cleavage. If the PAM site cannot be mutated, the alternative choice is to create several silent mutations within the gRNA recognition sequence. In addition, a transcription unit containing Ubc promoter and blasticidin-resisted gene, which is between two loxP sites in the same direction, was inserted in the intron regions to increase positive $\mathrm{KI}$ clones ratio. After blasticidin selection, a plasmid expressing Cre enzyme was transferred to cells to remove this transcription unit between two loxP sites.

3) Design and synthesize single-stranded donor oligonucleotides (ssODN) as repair template for LIG1-K126A KI cell line and MCM2-Y90A cell line.

For LIG1-K126A KI cell line and MCM2-Y90A cell line constriction, ssODN was used as repair template donor. The up arm and down arm are both $45 \mathrm{bp}$ in length. Nucleosides at K126 were changed to the ones coding alanine. Several mutations within the gRNA recognition sequence were introduced to prevent SpCas9 cleavage. At the same time, undesired amino acid changes were not introduced.

4) Design and construct miniAID (AID) targeting plasmids for UHRF1, CHAF1A and LSH inducible depletion cell line.

Plasmid based donor was used as repair template in AID targeting, and the homology arm is about $1 \mathrm{~kb}$ in length, too. The gRNA PAM site was mutated. AID tag was inserted immediately before the stop codon of targeted gene. A flexible GS linker is included between the terminus of gene and the AID tag to diminish the chance that the tag will affect the function of the endogenous gene. Puromycin resistant gene is appended after AID tag to increase positive $\mathrm{KI}$ clone ratio. ${ }^{62,63,90}$

5) DNMT3A/3B DKO cell line gRNA design and clone

Dual gRNAs were used to create frame-shift and generate KO cell line. gRNA was cloned to LentiCRISPR v2 as described above.

2. Transfection and antibiotic selection.

For DNMT1-H170V KI and UHRF1-TTD KI cell lines construction, two gRNA plasmids and one repair template donor plasmid were co-transfected to cells at the ratio of $1: 1: 1$ by mass using Lipofectamine 3000 reagent (Invitrogen, L3000) in a $3.5 \mathrm{~cm}$ dish. After transfection for 6-7 h, cells were washed with PBS for 3 times to remove transfection regent. Then cells were trypsin digested and transferred to $6 \mathrm{~cm}$ dish. $24 \mathrm{~h}$ after transfection, puromycin was added for 2 days for selection and then was removed by PBS washing. 6-7 days after transfection, blasticidin was added for more than 2 days. After several rounds of cell passage, transfected cells were seeded into 96-well dishes.

For LIG1-K126A, MCM2-Y90A KI, in which ssODN was used as repair template donor. $1.5 \mu \mathrm{g}$ LentiCRISPRv2 gRNA and $2 \mu \mathrm{L} 100$ $\mu \mathrm{M}$ ssODN were co-transferred to cells using Lipofectamine 3000 reagent in a $3.5 \mathrm{~cm}$ dish. After transfection for $6-7 \mathrm{~h}$, cells were washed with PBS for 3 times to remove transfection regent. Then cells were trypsin digested and transferred to $6 \mathrm{~cm}$ dish. $24 \mathrm{~h}$ after transfection, puromycin was added for 2 days for selection and then was removed by PBS washing. After several rounds of cell passage, transfected cells were seeded into 96-well dishes.

For UHRF1-AID, CHAF1A-AID, and LSH-AID inducible depletion cell lines construction, OsTIR1 stable expression cell line was firstly established. In which OsTIR1 gene (with Neo resistant, plasmid 
was modified from pMK232, Addgene \#72834) was inserted into AAVS1 site by CRISPR/Cas9 targeting. Secondly, AID-puro tag was targeted immediately before the stop codon of interested gene. Transfection and antibiotic selection of those two cell lines were similar to DNMT1-H170V KI and UHRF1-TTD KI cell lines construction.

For DNMT3A/3B DKO cell line, dual gRNAs were transfected at the ratio of $1: 1$ by mass using Lipofectamine 3000 reagent. DNMT3A KO cell line was established first, and then DNMT3B KO was generated based on DNMT3A KO cell line.

3) Genotyping to identify homozygous $\mathrm{KI}$ or KO clones

Single clones from the 96-well were picked and expanded. Homozygous $\mathrm{KI}$ and $\mathrm{KO}$ clones were identified by genotyping. The potential right clones were further validated at the RNA and/or protein levels by sequencing and/or western blot.

Haplotype information of HeLa cell line

Haplotype information of HeLa is available in the previous report.91 The detailed information about copy number of interested genes or interested regions can be obtained in the Supplementary information, Table S7 of this work.

Cell cycle analysis with flow cytometer

Cells were detached from dish by trypsin digestion, and washed with $1 \times$ PBS once. Add $0.5 \mathrm{~mL}$ ice-cold PBS to re-suspend cells. Add $5 \mathrm{~mL}-20^{\circ} \mathrm{C}$ pre-cold $70 \%$ ethanol and mix by pipetting. Put on ice for more than half one hour. Cells can be stored in $70 \%$ ethanol at $4{ }^{\circ} \mathrm{C}$ for several days. Centrifugation at $3600 \times g$ at $4{ }^{\circ} \mathrm{C}$ for $3 \mathrm{~min}$, remove supernatant. Re-suspend pellet with $5 \mathrm{~mL}$ icecold PBS and centrifuge at $3600 \times g$ at $4{ }^{\circ} \mathrm{C}$ for $3 \mathrm{~min}$. Remove supernatant. Repeat this step once to further remove remnant ethanol. Re-suspend cell pellet with $500 \mu \mathrm{L}$ Propidium iodide (PI) buffer and incubate at $37^{\circ} \mathrm{C}$ water bath for about $30 \mathrm{~min}$, keep from light. Every $1 \mathrm{~mL} \mathrm{PI}$ buffer contained $100 \mu \mathrm{l} 1 \mathrm{mg} / \mathrm{mL} \mathrm{PI}$ (Thermo Fisher, P3566), $60 \mu \mathrm{L} \mathrm{10 \%} \mathrm{TritonX-100,} 40 \mu \mathrm{L} 5 \mathrm{mg} / \mathrm{mL}$ RNaseA, $800 \mu \mathrm{L} 1 \times$ PBS. Samples are ready for flow cytometry analysis.

ChIP-seq

Add formaldehyde to cell culture medium to the final concentration of $1 \%$, crosslink at room temperature (RT) for 8-10 min. Add glycine to the final concentration of $0.125 \mathrm{M}$ to terminate crosslinking reaction. Wash in $1 \times$ PBS for three times, scrape and resuspend cells in $5 \mathrm{~mL}$ PBS. Centrifuge at $2000 \mathrm{rpm}$ at $4{ }^{\circ} \mathrm{C}$ for $3 \mathrm{~min}$. Remove PBS. Resuspend cells in $5 \mathrm{~mL}$ cell lysis buffer 1 (50 mM HEPES, pH 7.9, 140 mM NaCl, 1 mM EDTA, 10\% glycerol, $0.5 \%$ IGEPAL CA-630, 0.25\% TritonX-100) and incubate on ice for $10 \mathrm{~min}$. Centrifuge at $2000 \mathrm{rpm}$ at $4{ }^{\circ} \mathrm{C}$ for $5 \mathrm{~min}$. Remove supernatant. Resuspend cells in $5 \mathrm{~mL}$ cell lysis buffer $2(10 \mathrm{mM}$ Tris- $\mathrm{HCl}, \mathrm{pH} 8.0,200 \mathrm{mM} \mathrm{NaCl}, 1 \mathrm{mM}$ EDTA, $0.5 \mathrm{mM}$ EGTA) and incubate at room temperature for $10 \mathrm{~min}$. Centrifuge at $4000 \mathrm{rpm}$ at $4{ }^{\circ} \mathrm{C}$ for $5 \mathrm{~min}$. Remove supernatant. Add $1 \mathrm{~mL}$ cell lysis buffer 3 (10 mM Tris- $\mathrm{HCl}, \mathrm{pH} 8.0,100 \mathrm{mM} \mathrm{NaCl}, 1 \mathrm{mM}$ EDTA, $0.5 \mathrm{mM}$ EGTA, $0.1 \%$ Sodium deoxycholate, $0.5 \%$ Sodium laurate, $0.1 \%$ SDS, $1 \times$ proteinase inhibitor (Merck, 5892970001) and resuspend chromatin fraction. Put on ice. Sonicate chromatin to $200-400 \mathrm{bp}$ in average with Covaris M220. Centrifuge at $12,000 \mathrm{rpm}$ at $4^{\circ} \mathrm{C}$ for 10 min and transfer supernatant to a new $1.5 \mathrm{~mL}$ tube. Keep $10 \mu \mathrm{L}$ as "Input". Incubate chromatin fragments with antibody overnight at $4{ }^{\circ} \mathrm{C}$ with rotation. The next day, add pre-washed Dynabeads protein A (Thermo Fisher, 10002D) and/or Dynabeads protein G (Thermo Fisher, 10004D) to the tube and incubate for $3 \mathrm{~h}$ at $4{ }^{\circ} \mathrm{C}$ with rotation. The beads were sequentially washed in order as follows: $1 \mathrm{~mL}$ FA lysis buffer $(150 \mathrm{mM} \mathrm{NaCl}, 1 \%$ TritonX-100, $50 \mathrm{mM}$ HEPES, pH 7.9, 2 mM EDTA pH 8.0, 0.5\% sodium deoxycholate) once; $1 \mathrm{~mL} F A$ high salt buffer $(500 \mathrm{mM} \mathrm{NaCl}, 1 \%$ TritonX-100, $50 \mathrm{mM}$ HEPES, $\mathrm{pH} 7.9,2 \mathrm{mM}$ EDTA, $0.5 \%$ sodium deoxycholate) twice; $1 \mathrm{~mL} \mathrm{LiCl}$ buffer $(250 \mathrm{mM}$ LiCl, 0.5\% IGEPAL CA-630, 0.5\% sodium deoxycholate, $1 \mathrm{mM}$ EDTA, $10 \mathrm{mM}$ Tris- $\mathrm{HCl}$, $\mathrm{pH}$ 8.0) once; $1 \mathrm{~mL} 1 \times$ TE (10 mM Tris-HCl, pH 8.0 and $1 \mathrm{mM}$ EDTA) buffer for twice. Each washing last for 5 min with rotation at $4{ }^{\circ} \mathrm{C}$. Resuspend beads with $250 \mu \mathrm{L}$ ChIP elution buffer (1\% SDS, $100 \mathrm{mM} \mathrm{NaHCO}{ }_{3}$, freshly prepared) and incubate at room temperature with vortex for $15 \mathrm{~min}$. Transfer supernatant to a new tube. Repeat the elution step once and combine the elution fractions.

To reverse crosslinking, add $25 \mu \mathrm{L} 4 \mathrm{M} \mathrm{NaCl}$ to the $500 \mu \mathrm{L}$ elution fraction, incubate at $65^{\circ} \mathrm{C}$ for $8 \mathrm{~h}$. After reverse crosslinking, add $3 \mu \mathrm{L}$ $20 \mathrm{mg} / \mathrm{mL}$ RNaseA and incubate at $37^{\circ} \mathrm{C}$ for $30 \mathrm{~min}$. Add $20 \mu \mathrm{L} 1 \mathrm{M}$ Tris- $\mathrm{HCl}, \mathrm{pH} 6.5,10 \mu \mathrm{L} 0.5 \mathrm{M}$ EDTA, pH 8.0, and $7 \mu \mathrm{L} 20 \mathrm{mg} / \mathrm{mL}$ proteinase $\mathrm{K}$, incubate at $55^{\circ} \mathrm{C}$ for more than $4 \mathrm{~h}$. Extract DNA with phenol-chloroform method. Next generation sequencing library is prepared with KAPA Hyperprep Kit (KAPA, KK8504).

Whole genome bisulfite sequencing (WGBS) library preparation WGBS libraries of HeLa and mESC were prepared according to ${ }^{92}$

Data processing and analysis

Genome build. All analyses in this study were based on the human genome assembly GRCh37/hg19 for HeLa cells and mouse genome assembly $\mathrm{GRCm} 38 / \mathrm{mm} 10$ for mouse ES cells, respectively.

Hammer-seq data sequencing and processing. Raw reads from Illumina HiSeq $\times 10$ or NovaSeq 6000 platforms were processed by HBS-Tools. ${ }^{93}$ In brief, preprocessing of raw reads, including bad quality bases trimming, sequencing adapter, and hairpin adapter removal were performed with the module of "hbs_process". And then read 1 and read 2 of each pre-processed read pair were aligned pairwise using Smith-Waterman algorithm and only aligned parts were kept to recover the original genomic sequence. The recovered original sequences were then mapped to the genome assembly, with which the methylation information was extracted into the result SAM output. The original sequence recovery and mapping were performed with the module "hbs_mapper" of HBS-Tools.

The aligned SAM files, that contain both strands of the hairpin read, were then de-duplicated based on the following criteria: the two strands were firstly concatenated with the one with highermethylation as head and the lower as tail, and strand pairs with the same concatenated sequence were regarded as duplication and only one copy was retained.

Based on the de-duplicated aligned reads (in SAM file), reads of each pair were discriminated as parent (higher) or daughter (lower) strand according to the No. of methylated CpGs, with equally methylated reads randomly distributed to parent or daughter. Read pairs with no $\mathrm{CpGs}$ on both strands were discarded. Then, methylation maintenance events were called based on parent/daughter read pairs, among which maintained methylation, un-maintained methylation, un-methylation and de novo methylation were designated as $M M, M U, U U, U M$, respectively. All events were summed up for each genomic CpG site by chromosome coordinates, so now we have 4 numbers indicating 4 kinds of events of MM/MU/UU/UM for each CpG site.

Discriminated parent strand reads and daughter strand reads were used individually to calculate the methylation levels of parent and daughter strands using Bismark software. Only those CpG sites with coverage above 5 were used for calculation of average methylation. Data of WGBS of bulk HeLa cells and data of nascent EdU-IP-seq for newly synthesized DNA were processed with Bismark software similarly.

For CpG sites, only those sites with $(M M+M U) \geq 3$ were retained as covered sites and maintenance ratio was calculated as Ratio $=\mathrm{MM} /(\mathrm{MM}+\mathrm{MU})$.

Maintenance ratio of repeat elements and genomic regions. Gene features were extracted from annotations in UCSC genome browser. Enhancer annotation of HeLa cells were adopted from. ${ }^{94}$ 
Repeat elements were extract from 'rmsk' table of UCSC genome browser, and grouped by five classes. Maintenance ratios of CpG sites within each type of gnomic feature or repeat class were averaged to determine the maintenance kinetics (Fig. 1c, d).

De novo methylation frequency. For de novo methylation frequency, only those sites with $(U U+U M) \geq 5$ were regarded as covered sites and de novo methylation frequency was calculated as de novo $=\mathrm{UM} /(\mathrm{UM}+\mathrm{UU})$.

H3K9me2/H3K9me3/nucleosome occupancy and CpG density ChIP-seq reads for histone modifications (H3K9me2/H3K9me3, data generated in this study) and nucleosome occupancy (H3.1, adopt from GSM788634) were aligned to human genome assembly hg19 using Bowtie2 (v2.3.4.3), de-duplicated and normalized reads density within nearby $1 \mathrm{~kb}$ was assigned to each centered $\mathrm{CpG}$ site.

Similarly, No. of CpGs within nearby $100 \mathrm{bp}$ was assigned to the centered CpG site.

For neighboring $\mathrm{mCpG}$ density, methylation level (0-1) measured by HeLa WGBS (generated in this study) within neighboring $100 \mathrm{bp}$ of each CpG was summed up and assigned to the centered CpG site.

Division analysis

For division analysis (such as Fig. 1e), CpG sites were divided into 10 groups according to the ranges of feature of interest by 10 deciles (D1 range from decile of $0 \%$ to $10 \%, \ldots$, D10 range from decile of 90 to $100 \%$ ), and maintenance ratio of each division of CpG sites were averaged for plotting.

Leading/lagging strand analysis

OK-seq data were adopted from ${ }^{49}$ and RFD (replication fork direction) value for $1 \mathrm{~kb}$ bins were calculated as described. Bins with RFD above 0.6 or below -0.6 were kept and merged within 5,000 bps, respectively. The former merged regions were used as rightward replicating domains and the latter as leftward domains. Early/late replicating domains defined in this study were then intersected with rightward/leftward domains to get each type of left/right \& early/late domains.

Read 1 and read 2 of sequencing read pairs were firstly discriminated into parent (high) and daughter (low) strand by No. of methylation CpGs. Then read 1/2 were distributed into upper arm or lower arm of the replication fork according to the mapping strandedness of the recovered original sequences to the reference genome (see listed below, also illustrated by Fig. 1i).

\begin{tabular}{|c|c|c|c|c|}
\hline Fork arm & $\begin{array}{l}\text { R1 methyla- } \\
\text { tion }\end{array}$ & $\begin{array}{l}\text { R2 methyla- } \\
\text { tion }\end{array}$ & $\begin{array}{l}\text { Map to the } \\
\text { reference }\end{array}$ & $\begin{array}{l}\text { Position of hairpin } \\
\text { adapter }\end{array}$ \\
\hline Upper & High & Low & Positive strand & Right-ended \\
\hline Upper & Low & High & Negative strand & Left-ended \\
\hline Lower & Low & High & Positive strand & Right-ended \\
\hline Lower & High & Low & Negative strand & Left-ended \\
\hline
\end{tabular}

Read $1 / 2$ that possess the same number of methylated CpGs were randomly distributed into upper arm or lower arm.

So, the leading and lagging strand can be classified as shown in Fig. 1i, j. Reads located in upper arm and lower arm of leftward/ rightward replication domains were used to calculate the maintenance ratios as previously described, respectively.

Relative correlation for different bin sizes

To evaluate the effective range of neighboring CpGs (in Fig. 4f), CpG numbers in different sizes of bins for centered-CpG sites were correlated to the maintenance ratios. The sizes of bins were selected from $10 \mathrm{bp}, 20 \mathrm{bp}, \ldots$, to $250 \mathrm{bp}$, and the Pearson correlation coefficient was calculated for each size and plotted. All five chasing time points were plotted similarly. To easily compare the positions of summits, correlation coefficients were divided by the maximum value of every chasing time point (i.e., the max was normalized to 1).

Local CpG sequence contexts definition (solo-WCGW part) All covered CpG sites were grouped by 4-base context (WCGW/ SCGW/SCGS, WCGS was treated as SCGW) and number of flanking $\mathrm{CpGs}$, and mean maintenance ratio of each group of CpGs were plotted (Figs. 6b, 7f and Supplementary information, Fig. S6d). De novo methylation frequency was analyzed similarly (Fig. 6c).

Aging associated analysis

From 476, $366 \mathrm{CpG}$ sites examined in white blood cells from a population cohort by, ${ }^{68}$ we firstly select those 71,830 sites with $|\beta|$ $<2 \mathrm{E}-5$ as 'stable', then we selected those 137,618 sites that were significantly associated with aging $(P \leq 1.04 \mathrm{E}-7)$ and among that defined 54,698 sites with $\beta>0$ as 'increased', the other 82,920 with $\beta<0$ as 'decrease' (Fig. 6 d, e).

Statistical analysis

Slope of linear regression was calculated by $\mathrm{R}$, such as in Fig. 3a, and statistical analysis of two slopes were calculated according to Paternoster et al. ${ }^{95}$. Mann-Whitney U-test was used to calculate the significance of differences with $\mathrm{R}$ software as indicated in figure legends.

\section{DATA AVAILABILITY}

HeLa OK-seq data were obtained from NCBI SRA under the accession number SRP065949. ${ }^{49}$ Histone H3 ChIP-seq of HeLa S3 was obtained from GSM788634. ${ }^{96}$ Aging associated DNA methylation change data was taken from pulication. ${ }^{68}$ All high throughput data generated in this study have been deposited at NCBI GEO under accession number: GSE131098. Custom scripts for data analysis used in this data are available upon reasonable requests.

\section{ACKNOWLEDGEMENTS}

We thank J.Y. Jia and S. Sun for technical assistance in FACS analysis. This work was primarily supported by the National Natural Science Foundation of China (31530047). This work was also supported by the Ministry of Science and Technology of China (2016YFA0100400), the Chinese Academy of Sciences (XDB39000000 and QYZDYSSW-SMC031). Z.Z. is sponsored by the Youth Innovation Promotion Association (2017133) of the Chinese Academy of Sciences.

\section{AUTHOR CONTRIBUTIONS}

X.M., Z.Z. and B.Z. conceived and designed this project. X.M. performed the majority of experiments. Z.Z. performed bioinformatics analysis. Z.Zou., Q.H., Y.Y. helped in mutant cell line establishment. C.L. performed the UHPLC-MS/MS experiments under the supervision of H.W. Q.D. participated in experimental design and some experiments. Y.L. participated in data analysis. X.M., Z.Z. and B.Z. wrote the manuscript with comments and contributions from all authors.

\section{ADDITIONAL INFORMATION}

Supplementary information accompanies this paper at https://doi.org/10.1038/ s41422-020-0359-9.

Competing interests: The authors declare no competing interests.

\section{REFERENCES}

1. Jones, P. A. Functions of DNA methylation: islands, start sites, gene bodies and beyond. Nat. Rev. Genet. 13, 484-492 (2012).

2. Li, E. \& Zhang, Y. DNA methylation in mammals. Cold Spring Harb. Perspect. Biol. 6, a019133 (2014).

3. Bird, A. DNA methylation patterns and epigenetic memory. Genes Dev. 16, 6-21 (2002).

4. Araujo, F. D., Knox, J. D., Szyf, M., Price, G. B. \& Zannis-Hadjopoulos, M. Concurrent replication and methylation at mammalian origins of replication. Mol. Cell Biol. 18, 3475-3482 (1998). 
5. Gruenbaum, Y., Szyf, M., Cedar, H. \& Razin, A. Methylation of replicating and postreplicated mouse L-cell DNA. Proc. Natl. Acad. Sci. USA 80, 4919-4921 (1983).

6. Kappler, J. W. The kinetics of DNA methylation in cultures of a mouse adrenal cell line. J. Cell Physiol. 75, 21-31 (1970).

7. Xu, C. \& Corces, V. G. Nascent DNA methylome mapping reveals inheritance of hemimethylation at CTCF/cohesin sites. Science 359, 1166-1170 (2018).

8. Adams, R. L. The relationship between synthesis and methylation of DNA in mouse fibroblasts. Biochim Biophys. Acta 254, 205-212 (1971).

9. Liang, G. et al. Cooperativity between DNA methyltransferases in the maintenance methylation of repetitive elements. Mol. Cell Biol. 22, 480-491 (2002).

10. Woodcock, D. M. et al. Delayed DNA methylation is an integral feature of DNA replication in mammalian cells. Exp. Cell Res. 166, 103-112 (1986).

11. Charlton, J. et al. Global delay in nascent strand DNA methylation. Nat. Struct. Mol. Biol. 25, 327-332 (2018).

12. Bestor, T. H. The DNA methyltransferases of mammals. Hum. Mol. Genet. 9, 2395-2402 (2000).

13. Jeltsch, A. On the enzymatic properties of Dnmt1: specificity, processivity, mechanism of linear diffusion and allosteric regulation of the enzyme. Epigenetics 1, 63-66 (2006).

14. Song, J., Rechkoblit, O., Bestor, T. H. \& Patel, D. J. Structure of DNMT1-DNA complex reveals a role for autoinhibition in maintenance DNA methylation. Science 331, 1036-1040 (2011).

15. Song, J., Teplova, M., Ishibe-Murakami, S. \& Patel, D. J. Structure-based mechanistic insights into DNMT1-mediated maintenance DNA methylation. Science 335, 709-712 (2012).

16. Bashtrykov, P. et al. Specificity of Dnmt1 for methylation of hemimethylated CpG sites resides in its catalytic domain. Chem. Biol. 19, 572-578 (2012).

17. Bashtrykov, P., Ragozin, S. \& Jeltsch, A. Mechanistic details of the DNA recognition by the Dnmt1 DNA methyltransferase. FEBS Lett. 586, 1821-1823 (2012).

18. Fatemi, M., Hermann, A., Pradhan, S. \& Jeltsch, A. The activity of the murine DNA methyltransferase Dnmt1 is controlled by interaction of the catalytic domain with the N-terminal part of the enzyme leading to an allosteric activation of the enzyme after binding to methylated DNA. J. Mol. Biol. 309, 1189-1199 (2001).

19. Li, Y. et al. Stella safeguards the oocyte methylome by preventing de novo methylation mediated by DNMT1. Nature 564, 136-140 (2018).

20. Gowher, H. \& Jeltsch, A. Mammalian DNA methyltransferases: new discoveries and open questions. Biochem Soc. Trans. 46, 1191-1202 (2018).

21. Chuang, L. S. et al. Human DNA-(cytosine-5) methyltransferase-PCNA complex as a target for p21WAF1. Science 277, 1996-2000 (1997).

22. Schermelleh, L. et al. Dynamics of Dnmt1 interaction with the replication machinery and its role in postreplicative maintenance of DNA methylation. Nucleic Acids Res. 35, 4301-4312 (2007).

23. Spada, F. et al. DNMT1 but not its interaction with the replication machinery is required for maintenance of DNA methylation in human cells. J. Cell Biol. 176 565-571 (2007)

24. Xie, S. \& Qian, C. The growing complexity of UHRF1-mediated maintenance DNA methylation. Genes (Basel) 9, 600 (2018).

25. Karagianni, P., Amazit, L., Qin, J. \& Wong, J. ICBP90, a novel methyl K9 H3 binding protein linking protein ubiquitination with heterochromatin formation. Mol. Cell Biol. 28, 705-717 (2008).

26. Rothbart, S. B. et al. Association of UHRF1 with methylated H3K9 directs the maintenance of DNA methylation. Nat. Struct. Mol. Biol. 19, 1155-1160 (2012).

27. Zhao, Q. et al. Dissecting the precise role of $\mathrm{H} 3 \mathrm{~K} 9$ methylation in crosstalk with DNA maintenance methylation in mammals. Nat. Commun. 7, 12464 (2016).

28. Feinberg, A. P. \& Tycko, B. The history of cancer epigenetics. Nat. Rev. Cancer 4, 143-153 (2004)

29. Day, K. et al. Differential DNA methylation with age displays both common and dynamic features across human tissues that are influenced by CpG landscape. Genome Biol. 14, R102 (2013).

30. Heyn, H. et al. Distinct DNA methylomes of newborns and centenarians. Proc. Natl Acad. Sci. USA 109, 10522-10527 (2012).

31. Yuan, T. et al. An integrative multi-scale analysis of the dynamic DNA methylation landscape in aging. PLoS Genet. 11, e1004996 (2015).

32. Bollati, V. et al. Decline in genomic DNA methylation through aging in a cohort of elderly subjects. Mech. Ageing Dev. 130, 234-239 (2009).

33. Figueiredo, J. C. et al. Global DNA hypomethylation (LINE-1) in the normal colon and lifestyle characteristics and dietary and genetic factors. Cancer Epidemiol. Biomark. Prev. 18, 1041-1049 (2009).

34. Bocklandt, S. et al. Epigenetic predictor of age. PLoS One 6, e14821 (2011).

35. Horvath, S. DNA methylation age of human tissues and cell types. Genome Biol. 14, R115 (2013).

36. Hannum, G. et al. Genome-wide methylation profiles reveal quantitative views of human aging rates. Mol. Cell 49, 359-367 (2013).
37. Ciccarone, F., Tagliatesta, S., Caiafa, P. \& Zampieri, M. DNA methylation dynamics in aging: how far are we from understanding the mechanisms? Mech. Ageing Dev. 174, 3-17 (2018).

38. Field, A. E. et al. DNA methylation clocks in aging: categories, causes, and consequences. Mol. Cell 71, 882-895 (2018).

39. Klutstein, M., Nejman, D., Greenfield, R. \& Cedar, H. DNA methylation in cancer and aging. Cancer Res. 76, 3446-3450 (2016).

40. Zhou, W. et al. DNA methylation loss in late-replicating domains is linked to mitotic cell division. Nat. Genet. 50, 591-602 (2018).

41. Laird, C. D. et al. Hairpin-bisulfite PCR: assessing epigenetic methylation patterns on complementary strands of individual DNA molecules. Proc. Natl. Acad. Sci. USA 101, 204-209 (2004).

42. Zhao, L. et al. The dynamics of DNA methylation fidelity during mouse embryonic stem cell self-renewal and differentiation. Genome Res. 24, 1296-1307 (2014).

43. Jones, P. A. \& Baylin, S. B. The epigenomics of cancer. Cell 128, 683-692 (2007).

44. Hermann, A., Goyal, R. \& Jeltsch, A. The Dnmt1 DNA-(cytosine-C5)-methyltransferase methylates DNA processively with high preference for hemimethylated target sites. J. Biol. Chem. 279, 48350-48359 (2004).

45. Goyal, R., Reinhardt, R. \& Jeltsch, A. Accuracy of DNA methylation pattern preservation by the Dnmt1 methyltransferase. Nucleic Acids Res. 34, 1182-1188 (2006).

46. Vilkaitis, G., Suetake, I., Klimasauskas, S. \& Tajima, S. Processive methylation of hemimethylated $\mathrm{CpG}$ sites by mouse Dnmt1 DNA methyltransferase. J. Biol. Chem. 280, 64-72 (2005).

47. Petryk, N. et al. MCM2 promotes symmetric inheritance of modified histones during DNA replication. Science 361, 1389-1392 (2018).

48. $\mathrm{Yu}, \mathrm{C}$. et al. A mechanism for preventing asymmetric histone segregation onto replicating DNA strands. Science 361, 1386-1389 (2018).

49. Petryk, N. et al. Replication landscape of the human genome. Nat. Commun. 7, 10208 (2016).

50. Bostick, M. et al. UHRF1 plays a role in maintaining DNA methylation in mammalian cells. Science 317, 1760-1764 (2007).

51. Sharif, J. et al. The SRA protein Np95 mediates epigenetic inheritance by recruiting Dnmt1 to methylated DNA. Nature 450, 908-912 (2007).

52. Xie, S., Jakoncic, J. \& Qian, C. UHRF1 double tudor domain and the adjacent PHD finger act together to recognize K9me3-containing histone $\mathrm{H} 3$ tail. J. Mol. Biol. 415, 318-328 (2012).

53. Rothbart, S. B. et al. Multivalent histone engagement by the linked tandem Tudor and PHD domains of UHRF1 is required for the epigenetic inheritance of DNA methylation. Genes Dev. 27, 1288-1298 (2013).

54. Qin, W. et al. DNA methylation requires a DNMT1 ubiquitin interacting motif (UIM) and histone ubiquitination. Cell Res. 25, 911-929 (2015).

55. Rajakumara, E. et al. PHD finger recognition of unmodified histone H3R2 links UHRF1 to regulation of euchromatic gene expression. Mol. Cell 43, 275-284 (2011).

56. Nishiyama, A. et al. Uhrf1-dependent H3K23 ubiquitylation couples maintenance DNA methylation and replication. Nature 502, 249-253 (2013).

57. Ishiyama, S. et al. Structure of the Dnmt1 reader module complexed with a unique two-mono-ubiquitin mark on histone h3 reveals the basis for dna methylation maintenance. Mol. Cell 68, 350-360 e357 (2017).

58. Li, T. et al. Structural and mechanistic insights into UHRF1-mediated DNMT1 activation in the maintenance DNA methylation. Nucleic Acids Res. 46, 3218-3231 (2018).

59. Esteve, P. O. et al. Direct interaction between DNMT1 and G9a coordinates DNA and histone methylation during replication. Genes Dev. 20, 3089-3103 (2006).

60. Liu, X. et al. UHRF1 targets DNMT1 for DNA methylation through cooperative binding of hemi-methylated DNA and methylated H3K9. Nat. Commun. 4, 1563 (2013).

61. Ferry, L. et al. Methylation of DNA ligase 1 by G9a/GLP recruits UHRF1 to replicating DNA and regulates DNA methylation. Mol. Cell 67, 550-565 e555 (2017).

62. Lambrus, B. G., Moyer, T. C. \& Holland, A. J. Applying the auxin-inducible degradation system for rapid protein depletion in mammalian cells. Methods Cell Biol. 144, 107-135 (2018).

63. Nishimura, K., Fukagawa, T., Takisawa, H., Kakimoto, T. \& Kanemaki, M. An auxinbased degron system for the rapid depletion of proteins in nonplant cells. Nat. Methods 6, 917-922 (2009).

64. Mu, Z. M., Le, X. F., Vallian, S., Glassman, A. B. \& Chang, K. S. Stable overexpression of PML alters regulation of cell cycle progression in HeLa cells. Carcinogenesis 18, 2063-2069 (1997).

65. Kaufman, P. D., Kobayashi, R., Kessler, N. \& Stillman, B. The p150 and p60 subunits of chromatin assembly factor I: a molecular link between newly synthesized histones and DNA replication. Cell 81, 1105-1114 (1995).

66. Huang, $H$. et al. A unique binding mode enables MCM2 to chaperone histones H3-H4 at replication forks. Nat. Struct. Mol. Biol. 22, 618-626 (2015). 
67. Gan, H. et al. The Mcm2-Ctf4-polalpha axis facilitates parental histone $\mathrm{H} 3-\mathrm{H} 4$ transfer to lagging strands. Mol. Cell 72, 140-151 e143 (2018).

68. Johansson, A., Enroth, S. \& Gyllensten, U. Continuous aging of the human DNA Methylome throughout the human lifespan. PLoS One 8, e67378 (2013).

69. Dmitrijeva, M., Ossowski, S., Serrano, L. \& Schaefer, M. H. Tissue-specific DNA methylation loss during ageing and carcinogenesis is linked to chromosome structure, replication timing and cell division rates. Nucleic Acids Res. 46, 7022-7039 (2018).

70. Hon, G. C. et al. Global DNA hypomethylation coupled to repressive chromatin domain formation and gene silencing in breast cancer. Genome Res. 22, 246-258 (2012).

71. Berman, B. P. et al. Regions of focal DNA hypermethylation and long-range hypomethylation in colorectal cancer coincide with nuclear lamina-associated domains. Nat. Genet. 44, 40-46 (2011).

72. Costantini, M. \& Bernardi, G. Replication timing, chromosomal bands, and isochores. Proc. Natl. Acad. Sci. USA 105, 3433-3437 (2008).

73. Dennis, K., Fan, T., Geiman, T., Yan, Q. \& Muegge, K. Lsh, a member of the SNF2 family, is required for genome-wide methylation. Genes Dev. 15, 2940-2944 (2001).

74. Eckhardt, F. et al. DNA methylation profiling of human chromosomes 6, 20 and 22. Nat. Genet. 38, 1378-1385 (2006).

75. Jackson, D. A. \& Pombo, A. Replicon clusters are stable units of chromosome structure: evidence that nuclear organization contributes to the efficient activation and propagation of S phase in human cells. J. Cell Biol. 140, 1285-1295 (1998).

76. Sogo, J. M., Stahl, H., Koller, T. \& Knippers, R. Structure of replicating simian virus 40 minichromosomes. The replication fork, core histone segregation and terminal structures. J. Mol. Biol. 189, 189-204 (1986).

77. Casillas, M. A. Jr, Lopatina, N., Andrews, L. G. \& Tollefsbol, T. O. Transcriptional control of the DNA methyltransferases is altered in aging and neoplasticallytransformed human fibroblasts. Mol. Cell Biochem 252, 33-43 (2003).

78. Ronn, T. et al. Impact of age, BMI and $\mathrm{HbA} 1 \mathrm{c}$ levels on the genome-wide DNA methylation and mRNA expression patterns in human adipose tissue and identification of epigenetic biomarkers in blood. Hum. Mol. Genet. 24, 3792-3813 (2015).

79. Valentini, E. et al. Analysis of the machinery and intermediates of the 5hmCmediated DNA demethylation pathway in aging on samples from the MARK-AGE Study. Aging (Albany NY) 8, 1896-1922 (2016).
80. Berger S. L., Sassone-Corsi P. Metabolic signaling to chromatin. Cold Spring Harb. Perspect. Biol. 8, a019463 (2016).

81. Alisch, R. S. et al. Age-associated DNA methylation in pediatric populations. Genome Res. 22, 623-632 (2012).

82. Shipony, Z. et al. Dynamic and static maintenance of epigenetic memory in pluripotent and somatic cells. Nature 513, 115-119 (2014).

83. Levine, M. E. et al. An epigenetic biomarker of aging for lifespan and healthspan. Aging (Albany NY) 10, 573-591 (2018).

84. De Cecco, M. et al. L1 drives IFN in senescent cells and promotes age-associated inflammation. Nature 566, 73-78 (2019).

85. Presolski, S. I., Hong, V. P. \& Finn, M. G. Copper-catalyzed azide-alkyne click chemistry for bioconjugation. Curr. Protoc. Chem. Biol. 3, 153-162 (2011).

86. Ramachandran, S. \& Henikoff, S. Transcriptional regulators compete with nucleosomes post-replication. Cell 165, 580-592 (2016).

87. Sanjana, N. E., Shalem, O. \& Zhang, F. Improved vectors and genome-wide libraries for CRISPR screening. Nat. Methods 11, 783-784 (2014).

88. Haeussler, M. et al. Evaluation of off-target and on-target scoring algorithms and integration into the guide RNA selection tool CRISPOR. Genome Biol. 17, 148 (2016).

89. $\mathrm{Xu}, \mathrm{H}$. et al. Sequence determinants of improved CRISPR sgRNA design. Genome Res. 25, 1147-1157 (2015).

90. Natsume, T., Kiyomitsu, T., Saga, Y. \& Kanemaki, M. T. Rapid protein depletion in human cells by auxin-inducible degron tagging with short homology donors. Cell Rep. 15, 210-218 (2016).

91. Adey, A. et al. The haplotype-resolved genome and epigenome of the aneuploid HeLa cancer cell line. Nature 500, 207-211 (2013).

92. Urich, M. A., Nery, J. R., Lister, R., Schmitz, R. J. \& Ecker, J. R. MethylC-seq library preparation for base-resolution whole-genome bisulfite sequencing. Nat. Protoc. 10, 475-483 (2015).

93. Sun, M. A., Velmurugan, K. R., Keimig, D. \& Xie, H. HBS-tools for hairpin bisulfite sequencing data processing and analysis. Adv. Bioinforma. 2015, 760423 (2015).

94. Heintzman, N. D. et al. Histone modifications at human enhancers reflect global cell-type-specific gene expression. Nature 459, 108-112 (2009).

95. Paternoster, R., Brame, R., Mazerolle, P. \& Piquero, A. Using the correct statistical test for the equality of regression coefficients. Criminology 36, 859-866 (1998).

96. Ray-Gallet, D. et al. Dynamics of histone H3 deposition in vivo reveal a nucleosome gap-filling mechanism for H3.3 to maintain chromatin integrity. Mol. Cell 44, 928-941 (2011). 